\title{
Giant dipole resonance built on hot rotating nuclei produced during evaporation of light particles from the ${ }^{88} \mathrm{Mo}$ compound nucleus
}

M. Ciemała, ${ }^{1,2,{ }^{*}}$ M. Kmiecik, ${ }^{1}$ A. Maj, ${ }^{1}$ K. Mazurek, ${ }^{1}$ A. Bracco,${ }^{3}$ V. L. Kravchuk, ${ }^{4,5}$ G. Casini, ${ }^{6}$ S. Barlini, ${ }^{7}$ G. Baiocco ${ }^{8}$ L. Bardelli, ${ }^{7}$ P. Bednarczyk, ${ }^{1}$ G. Benzoni, ${ }^{9}$ M. Bini, ${ }^{7}$ N. Blasi, ${ }^{9}$ S. Brambilla,,${ }^{9}$ M. Bruno, ${ }^{8}$ F. Camera, ${ }^{3}$ S. Carboni, ${ }^{7}$ M. Cinausero, ${ }_{4}^{4}$ A. Chbihi, ${ }^{2}$ M. Chiari, ${ }^{7}$ A. Corsi, ${ }^{3}$ F. C. L. Crespi, ${ }^{3}$ M. D'Agostino, ${ }^{8}$ M. Degerlier, ${ }^{4}$ B. Fornal, ${ }^{1}$ A. Giaz, ${ }^{3}$ F. Gramegna, ${ }^{4}$ M. Krzysiek, ${ }^{1}$ S. Leoni, ${ }^{3}$ T. Marchi, ${ }^{4}$ M. Matejska-Minda, ${ }^{1,}{ }^{10}$ I. Mazumdar, ${ }^{11}$ W. Męczyński, ${ }^{1}$ B. Million, ${ }^{9}$ D. Montanari, ${ }^{3}$ L. Morelli, ${ }^{8}$ S. Myalski, ${ }^{1}$ A. Nannini, ${ }^{7}$ R. Nicolini, ${ }^{3}$ G. Pasquali, ${ }^{7}$ S. Piantelli, ${ }^{6}$ G. Prete, ${ }^{4}$ O. J. Roberts, ${ }^{12}$ Ch. Schmitt, ${ }^{2}$ J. Styczeń, ${ }^{1}$ B. Szpak, ${ }^{1}$ S. Valdré, ${ }^{7}$ B. Wasilewska, ${ }^{1}$ O. Wieland, ${ }^{9}$ J. P. Wieleczko, ${ }^{2}$ M. Ziębliński, ${ }^{1}$ J. Dudek, ${ }^{13}$ and N. Dinh Dang ${ }^{14}$

${ }^{1}$ Institute of Nuclear Physics Polish Academy of Sciences, 31-342 Kraków, Poland

${ }^{2}$ Grand Accélérateur National d'Ions Lourds (GANIL), B.P. 55027, F-14076 Caen Cedex 5, France

${ }^{3}$ Dipartimento di Fisica, Universitá di Milano and INFN Sezione di Milano, I-20133 Milano, Italy

${ }^{4}$ INFN, Laboratori Nazionali di Legnaro, I-35020 Legnaro, Italy

${ }^{5}$ National Research Centre “Kurchatov Institute”, 123182 Moscow, Russia

${ }^{6}$ INFN Sezione di Firenze, I-50019 Sesto Fiorentino, Italy

${ }^{7}$ Dipartimento di Fisica, Universitá di Firenze and INFN Sezione di Firenze, I-50019 Sesto Fiorentino, Italy

${ }^{8}$ Dipartimento di Fisica e Astronomia, Universitá di Bologna and INFN Sezione di Bologna, I-40127 Bologna, Italy

${ }^{9}$ INFN Sezione di Milano, I-20133 Milano, Italy

${ }^{10}$ Heavy Ion Laboratory, University of Warsaw, 02-093 Warsaw, Poland

${ }^{11}$ Tata Institute of Fundamental Research, 400005 Mumbai, India

${ }^{12}$ University of York, Heslington, YO10 5DD York, United Kingdom

${ }^{13}$ Institut Pluridisciplinaire Hubert Curien and Université de Strasbourg, B.P. 28, F-67037 Strasbourg Cedex 2, France

${ }^{14}$ Nishina Center for Accelerator-Based Science, RIKEN, Wako City, 351-0198 Saitama, Japan and Institute for Nuclear Science and Technique, Hanoi, Vietnam

(Received 27 February 2015; revised manuscript received 16 April 2015; published 13 May 2015)

\begin{abstract}
High-energy giant dipole resonance (GDR) $\gamma$ rays were measured following the decay of the hot, rotating compound nucleus of ${ }^{88} \mathrm{Mo}$, produced at excitation energies of 124 and $261 \mathrm{MeV}$. The reaction ${ }^{48} \mathrm{Ti}+{ }^{40} \mathrm{Ca}$ at 300 and $600 \mathrm{MeV}$ bombarding energies has been used. The data were analyzed using the statistical model Monte Carlo code GEMINI ++ . It allowed extracting the giant dipole resonance parameters by fitting the high-energy $\gamma$-ray spectra. The extracted GDR widths were compared with the available data at lower excitation energy and with theoretical predictions based on (i) The Lublin-Strasbourg drop macroscopic model, supplemented with thermal shape fluctuations analysis, and (ii) The phonon damping model. The theoretical predictions were convoluted with the population matrices of evaporated nuclei from the statistical model GEMINI++. Also a comparison with the results of a phenomenological expression based on the existing systematics, mainly for lower temperature data, is presented and discussed. A possible onset of a saturation of the GDR width was observed around $T=3 \mathrm{MeV}$.
\end{abstract}

DOI: 10.1103/PhysRevC.91.054313

PACS number(s): 21.10.Re, 24.30.Cz, 25.70.Gh, 27.50.+e

\section{INTRODUCTION}

Studying the giant dipole resonance (GDR) properties at high temperature and increasing angular momentum can be seen as one of the most important tools to investigate the nuclear structure under extreme conditions. In particular, the evolution of the GDR width with angular momentum and temperature reflects the role played by quantal and thermal fluctuations in the mechanism of damping of the giant resonance [1-7].

The GDR width has been measured for several nuclei and at various temperatures in the past. For the low-temperature range, say, $T<1.5 \mathrm{MeV}$, where in some cases shell effects play an important role, many results are available (see, e.g., [8-11]). Results show that at low excitation energies the GDR

*michal.ciemala@ifj.edu.pl width is nearly constant, whereas above a certain "critical" temperature, an increase is observed. This observation is rather systematic and rather well described by calculations taking into account not only the thermal shape fluctuations and shell corrections but also the quadrupole moment induced by the GDR at low temperatures. For the highest temperature region, up to about $3.7 \mathrm{MeV}$, the latest results on the ${ }^{132} \mathrm{Ce}$ nucleus show an almost linear increase of the GDR width as a function of temperature [12].

The observed dependence of the GDR width on the nuclear temperature was found to be mainly due to the increase in nuclear deformation and, at the higher temperatures (above $2.5 \mathrm{MeV}$ ), also due to the lifetime of the compound nucleus. In addition, it was shown that it is very important to properly evaluate the excitation energy of the compound nucleus since pre-equilibrium processes may occur at high bombarding energies [13]. Exclusive measurements were also made to address the problem of isospin mixing for $A=80$ nuclei [14]. 
It is clear from the present knowledge that more studies of the GDR properties at high temperature in a wide mass range are necessary to test predictions of damping mechanisms in a more comprehensive way. These studies should be based on exclusive and rather complete measurements which include, in addition to $\gamma$ rays, the detection of the recoiling residual nuclei and emitted particles.

The present article reports on an exclusive experiment performed to measure the GDR width of the ${ }^{88}$ Mo nucleus at high temperatures. The fusion-evaporation reaction ${ }^{48} \mathrm{Ti}+{ }^{40} \mathrm{Ca}$ was used and the experimental setup allowed the detection of the recoiling residual nuclei and the charged particles together with high-energy $\gamma$ rays. For Mo isotopes, the GDR width data exist already in the literature at lower temperatures. The results presented here are essential to give a more complete picture of the behavior of the GDR width as a function of the nuclear temperature and of GDR damping mechanisms.

\section{EXPERIMENT}

The decay of compound ${ }^{88}$ Mo nuclei was measured in an experiment performed at the TANDEM+ALPI accelerator of Legnaro National Laboratories, Legnaro, Italy. Beams of ${ }^{48} \mathrm{Ti}$ ions with bombarding energies of 300 and $600 \mathrm{MeV}$ in the laboratory system impinged on a $500 \mu \mathrm{g} / \mathrm{cm}^{2}{ }^{40} \mathrm{Ca}$ target. The beam was pulsed by the bunching system and the time between ca. 1 ns wide bunches was 200 ns. The compound nuclei $(\mathrm{CN})$ were produced at excitation energies of 124 and $261 \mathrm{MeV}$, corresponding to the maximal $\mathrm{CN}$ temperature of 3.2 and $4.8 \mathrm{MeV}$, respectively. The fusion cross section, calculated with the Bass model $[15,16]$ was 1.32 barns at a bombarding energy of $300 \mathrm{MeV}$, and 0.75 barns at $600 \mathrm{MeV}$. The maximum values of the transferred angular momentum reached, respectively $78 \hbar$ and $84 \hbar$, both values exceeding the value of $64 \hbar$ corresponding to the spin at which the fission barrier of ${ }^{88} \mathrm{Mo}$ vanishes. The relevant reaction parameters, such as the fusion cross section, cross section for the evaporation residues, $\mathrm{CN}$ velocity, excitation energy, and temperature for both beam energies, are listed in Table I.

A combination of three detector systems was employed during this experiment (see Fig. 1). The GARFIELD array [17,18] was used to detect light charged particles (LCP) and light reaction fragments whereas the HECTOR setup [19] detected high-energy $\gamma$ rays. Finally, a set of phoswich detectors [20] was employed to detect the charged nuclear products in a

TABLE I. Parameters of the ${ }^{48} \mathrm{Ti}+{ }^{40} \mathrm{Ca}$ reaction, where $E_{\mathrm{LAB}}^{b}$ denotes beam energy; $v_{\mathrm{CN}} / c$ : velocity of compound nucleus divided by speed of light; $\mathrm{CN} E_{\mathrm{x}}$ : $\mathrm{CN}$ excitation energy; $T_{\mathrm{CN}}^{\max }$ : maximum $\mathrm{CN}$ temperature; $\sigma_{\text {fus }}:$ cross-section for fusion; $\sigma_{\mathrm{ER}}$ : cross-section for the evaporation residues; and $l_{\max }$ : the maximum angular momentum brought to the nucleus.

\begin{tabular}{lccccccc}
\hline \hline $\begin{array}{l}E_{\mathrm{LAB}}^{b} \\
(\mathrm{MeV})\end{array}$ & $\begin{array}{c}v_{\mathrm{CN}} / c \\
(\%)\end{array}$ & $\begin{array}{c}\mathrm{CN} E_{\mathrm{x}} \\
(\mathrm{MeV})\end{array}$ & $\begin{array}{c}T_{\mathrm{CN}}^{\max } \\
(\mathrm{MeV})\end{array}$ & $\begin{array}{c}\sigma_{\text {fus }} \\
(\text { barn })\end{array}$ & $\begin{array}{c}\sigma_{\mathrm{ER}} \\
(\text { barn })\end{array}$ & $\begin{array}{c}\frac{\sigma_{\mathrm{ER}}}{\sigma_{\text {fus }}} \\
(\%)\end{array}$ & $\begin{array}{c}l_{\max } \\
(\hbar)\end{array}$ \\
\hline 300 & 6.27 & 123.8 & 3.2 & 1.32 & 0.53 & 40 & 78 \\
600 & 8.91 & 260.7 & 4.8 & 0.76 & 0.15 & 20 & 84 \\
\hline \hline
\end{tabular}

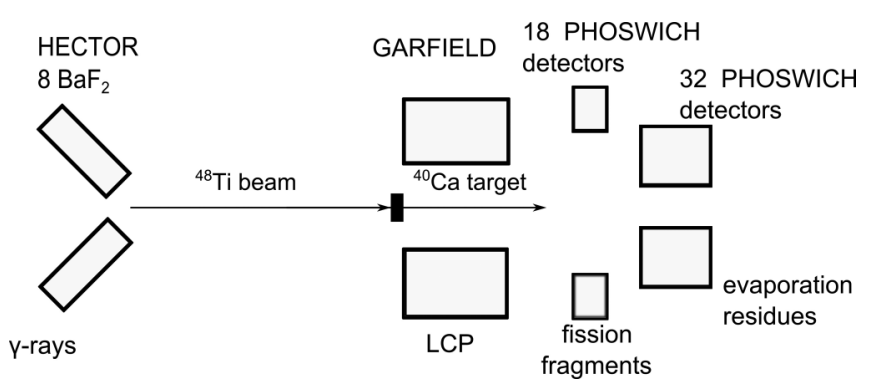

FIG. 1. Schematic view of the experimental setup in the ${ }^{48} \mathrm{Ti}+{ }^{40} \mathrm{Ca} \rightarrow{ }^{88} \mathrm{Mo} *$ experiment.

very broad mass area, ranging from light charged particles to evaporation residues (ER).

The GARFIELD array is composed of a drift chamber divided azimuthally into 24 sectors, each of them consisting of $8 \Delta E-E$ telescopes. The $\mathrm{CsI}(\mathrm{Tl})$ scintillation crystals placed in the same gas volume, are used to measure the total energy $(E)$ of the light charged particles (LCP). During experiment, the forward part of the GARFIELD apparatus was used, covering $\theta=29^{\circ}$ to $\theta=82^{\circ}$ and $2 \pi$ in $\phi$ angles. Figure 2 shows the fast and slow components from the $\mathrm{CsI}(\mathrm{Tl})$ detectors which were used to select alpha and proton decays.

The HECTOR array consisting of 8 large volume $(14.5 \times 17 \mathrm{~cm}) \mathrm{BaF}_{2}$ scintillation crystals was placed at backward angles $\left(\Theta\right.$ from $125^{\circ}$ to $\left.160^{\circ}\right)$ with respect to the beam direction. The detectors were positioned inside of the GARFIELD vacuum chamber at a distance of $30 \mathrm{~cm}$ from the target. This distance was chosen as a compromise between detection efficiency and a sufficiently long flight path for the neutron discrimination via the time-of-flight method. The energy calibration of the $\mathrm{BaF}_{2}$ scintillators was performed using ${ }^{137} \mathrm{Cs},{ }^{60} \mathrm{Co}$, and ${ }^{88} \mathrm{Y}$ sources for the low-energy interval, and $15.1 \mathrm{MeV} \gamma$ rays from the ${ }^{11} \mathrm{~B}+{ }^{2} \mathrm{H} \rightarrow{ }^{12} \mathrm{C}+n$ reaction at $19 \mathrm{MeV}$. During the experiment, the electronic threshold was set at a value corresponding to the $\gamma$ rays of 4.5 $\mathrm{MeV}$ and thus the rejection of low-energy $\gamma$ rays reduced the dead-time of the data acquisition system.

The phoswich detectors consisted of two layers of $180 \mu \mathrm{m}$ and $5 \mathrm{~mm}$ thick plastic scintillators backed by a $4 \mathrm{~cm}$ thick

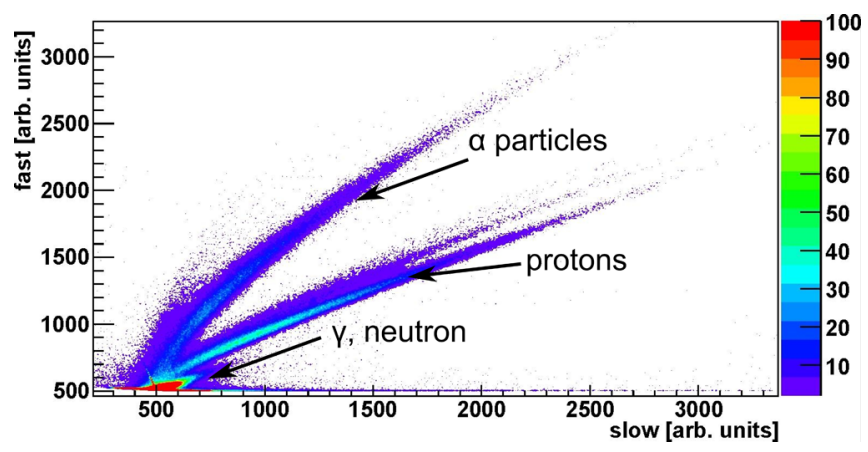

FIG. 2. (Color online) Correlations between the fast and slow components of $\mathrm{CsI}(\mathrm{Tl})$ scintillation signal. The $2 \mathrm{D}$ plot shows a very good separation of protons and alpha-particle event distributions. The data are from the ${ }^{48} \mathrm{Ti}+{ }^{40} \mathrm{Ca}$ reaction at the bombarding energy of $300 \mathrm{MeV}$. 


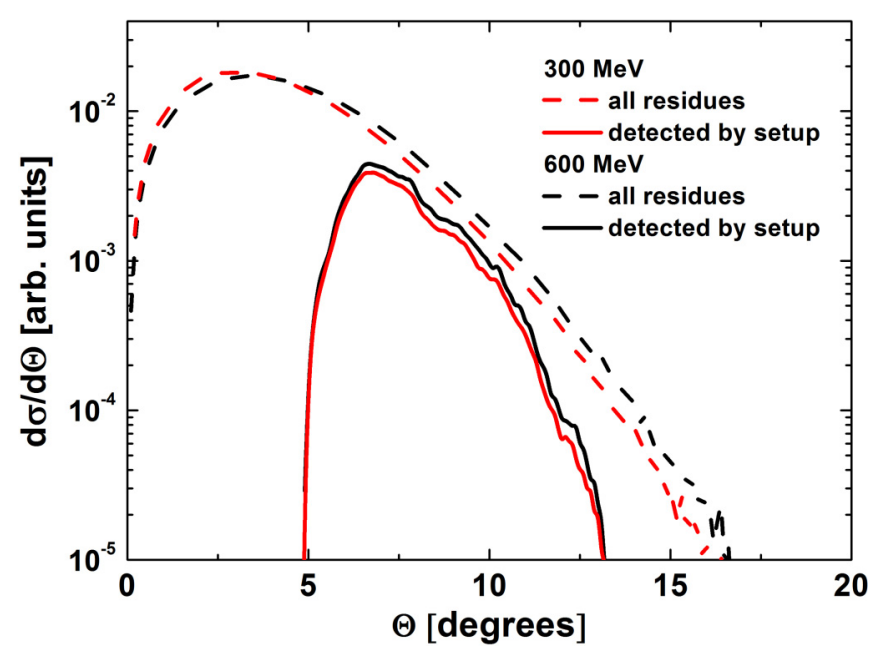

FIG. 3. (Color online) Angular distribution (in the laboratory) of the evaporation residues at $300 \mathrm{MeV}$ (red) and $600 \mathrm{MeV}$ (black) beam energy. Dashed lines represent emitted residues (GEMINI++ calculations), while continuous lines correspond to the residues which fulfill experimental detection conditions (GEMINI++ calculations with event-by-event filtering when residue is entering phoswich detectors).

$\mathrm{CsI}(\mathrm{Tl})$ crystal. For each phoswich three energy related signals were obtained: $g A$ dentoes the fastest light components from the three scintillator layers, $g B$ the light of the second plastic layer and part of light emitted by $\operatorname{CsI}(\mathrm{Tl})$, and $g C$ the light emitted by $\mathrm{CsI}(\mathrm{Tl})$. The phoswich detectors were positioned around the beam at forward angles $\left(\Theta\right.$ from $5^{\circ}$ to $13^{\circ}$, corresponding to $0.1 \mathrm{sr}$ ) at $16 \mathrm{~cm}$ distance from the target. Figure 3 shows selected regions of the angular distributions of evaporation residues produced in the reactions considered in this paper. As the limited angular acceptance of the evaporation residua might distorted the spectra of emitted charged particles, in all subsequent statistical model calculations such experimental conditions were taken into account.

The evaporation-residue selection was done by gating on the $g A$ energy and on the time of flight (ToF) of the residues (see Fig. 4, top) obtained in respect to the RF signal from the beam bunching system. Time 0 has been fixed as the time when beam hits the target, based on elastic scattering and known geometry of the detectors. The correctness of the evaporation residue selection was checked by inspecting Fig. 4, bottom, which shows the $\gamma$-ray energy registered by HECTOR versus the time of flight of the heavy fragments. High-energy $\gamma$ rays $\left(E_{\gamma}>10 \mathrm{MeV}\right)$ were observed in the events corresponding to the emission from the evaporation residues (ToF interval between 70 and $110 \mathrm{~ns}$ ). The total residue detection efficiency, after taking into account the solid angle (Fig. 3) and gating conditions (Fig. 4) was ca. $12 \%$.

All analyzed data were collected with the logical condition of coincidence: [OR of signals from phoswich detectors] AND [OR of signals above threshold from HECTOR array]. An additional gate was used to remove the neutron events in the $\gamma$-ray spectra. This gate was set on the time of flight (ToF) measured with the $\mathrm{BaF}_{2}$ HECTOR detectors in respect to the
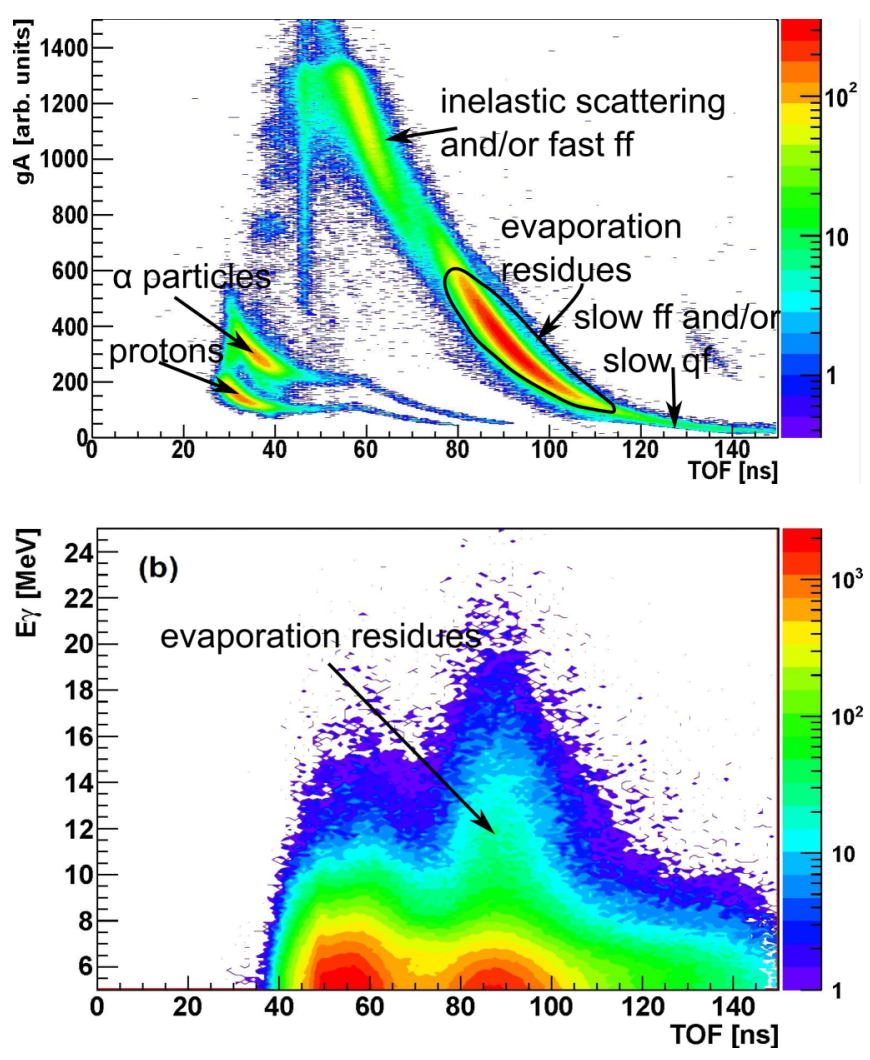

FIG. 4. (Color online) (a) 2D plot of the $g A$ parameter $(\Delta E$ energy deposit) and ToF (time of flight) dependence for one of the 32 phoswich detectors, with indicated regions for different reaction products [e.g., particles, evaporation residues, fission fragments (f)f, quasifission fragments (qf), etc.]. (b) 2D plot of the $\gamma$-ray energy $\left(E_{\gamma}\right)$ in HECTOR versus the ToF for heavy fragments in the phoswich detector. The data are for the $300 \mathrm{MeV}$ reaction.

RF signal from the bunching system. Here time 0 was set as the time of arrival of the prompt gamma rays from the target to the detector. An example of such a ToF spectrum measured at the beam energy of $300 \mathrm{MeV}$ is shown in Fig. 5 .

As it can clearly be seen from the figure, neutrons arrive a few ns after the $\gamma$ rays. Additionally, the contributions of the Bremsstrahlung and cosmic rays were subtracted from the $\gamma$-ray spectrum. This contribution was estimated by fitting the exponential functions to the $\gamma$-ray energy spectra over the interval of 30-35 MeV. In Fig. 6, $\gamma$-ray spectra, before and after the application of these gates are presented, as described above.

In addition to the high-energy $\gamma$-rays, the energy of the charged particles was measured. A careful analysis of the evaporated light charged particles (LCP) together with a comparison to the statistical model GEMINI++ of [21] is reported in a specifically devoted article [22]. For the current analysis, the output of GEMINI++ Monte Carlo statistical model calculations (described in the next chapter) was sorted using the same conditions as for sorting the experimental data.

All the conditions characterizing the experimental data were taken into account in the calculations, including the experimental acceptances of the GARFIELD and phoswich detectors. Such requirements enable the normalization of the 


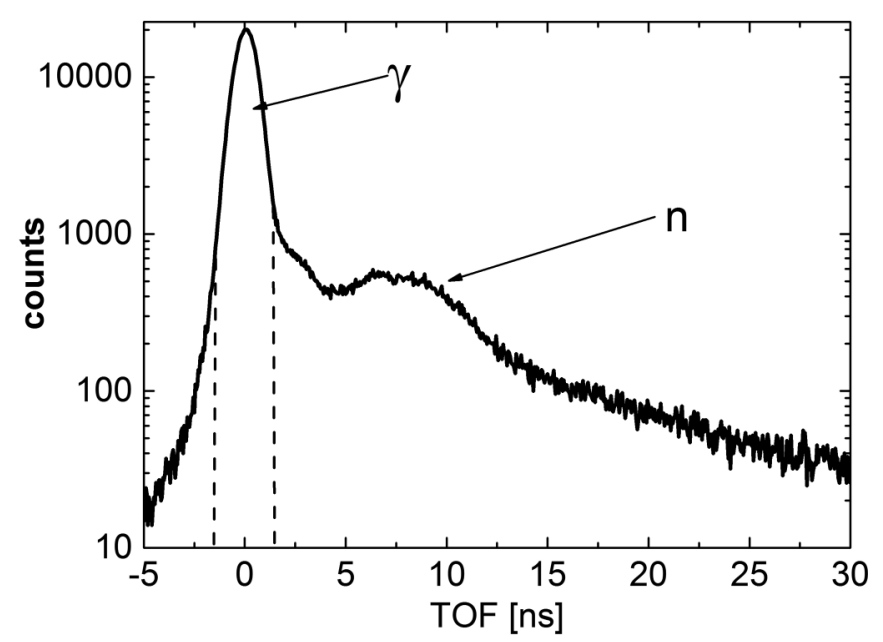

FIG. 5. Time-of-flight (ToF) spectrum for the HECTOR detectors, measured for the ${ }^{48} \mathrm{Ti}+{ }^{40} \mathrm{Ca}$ reaction, at the beam energy of $300 \mathrm{MeV}$. Dashed lines define the gating region for $\gamma$-ray selection.

LCP spectra, the measured and the calculated ones, using the number of registered particles. The experimental spectra, as shown in Fig. 7, are in a rather good agreement with the statistical model calculations. Only in the case of the alpha spectrum for the $600 \mathrm{MeV}$ bombarding energy one finds a discrepancy which might be attributed to a smaller detector efficiency at highest energies of the $\alpha$ particles. Figure 7 demonstrates that the statistical evaporation code GEMINI++ is capable of describing the charged-particle emission even at the very high excitation energies considered here.

Let us emphasize at this point that the detection of the LCP (protons and $\alpha$ particles) is important because it may provide a signal of a possible contribution of pre-equilibrium emission. Such a contribution would be visible as an additional highenergy shoulder in the energy spectra (see Fig. 1 in Ref. [12]). If

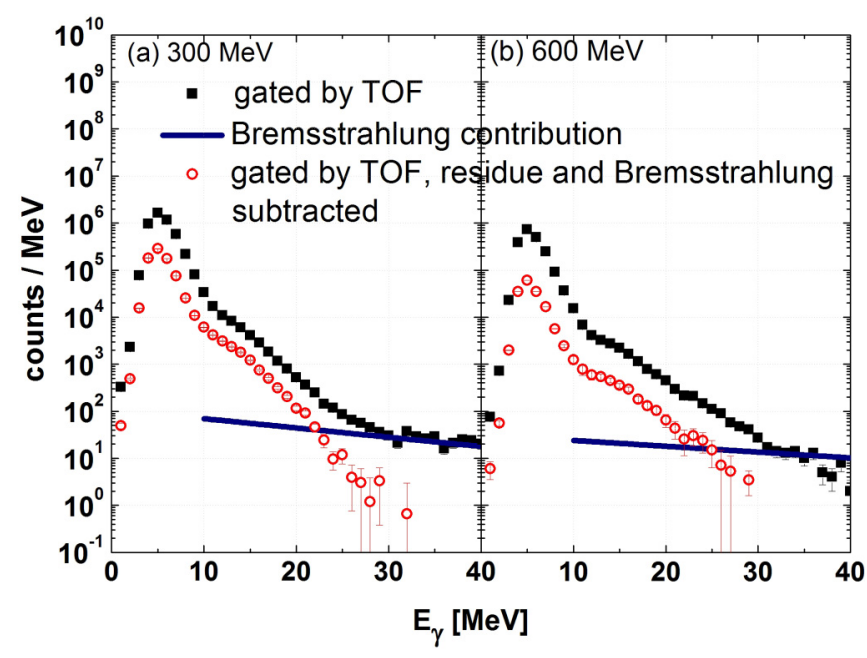

FIG. 6. (Color online) Measured $\gamma$-ray energy-spectra obtained while gating on the HECTOR ToF (full squares) and on the residues after subtracting the cosmic-ray and Bremsstrahlung contributions (open circles); (a) $300 \mathrm{MeV}$ data, (b) $600 \mathrm{MeV}$ data.

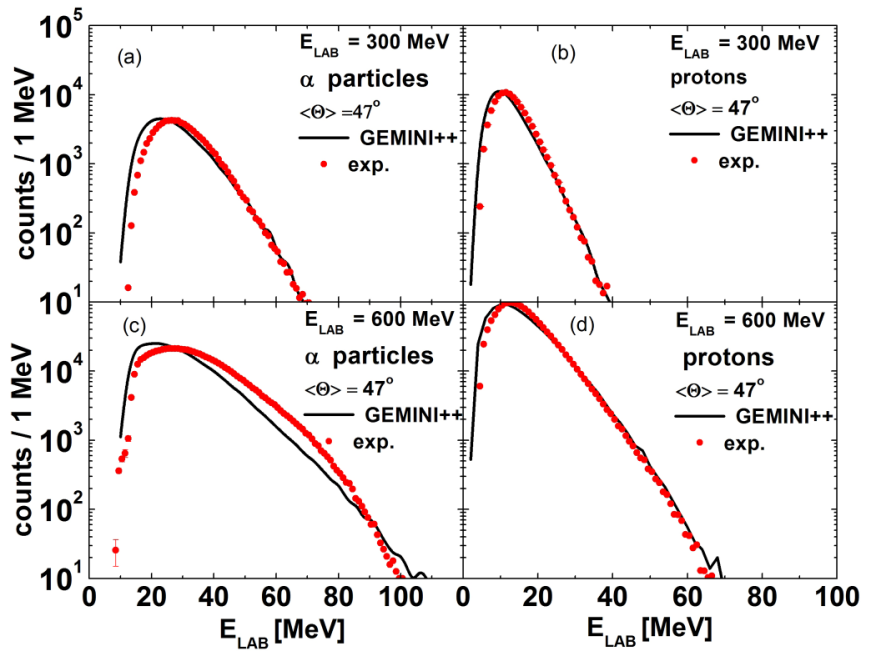

FIG. 7. (Color online) The experimental alpha-particle (left) and proton (right) energy spectra for the beam energies of $300 \mathrm{MeV}$ (top) and $600 \mathrm{MeV}$ (bottom); full circles. They correspond to measurements with the detectors placed at the mean angle of $\theta=$ $47^{\circ}$. The full lines show the results of the statistical code GEMINI++ model calculations.

such contribution is present, the fusion is not complete and the formed system is not produced at the expected values of spin and excitation energy. Our study demonstrated that the preequilibrium is almost absent even at the highest bombarding energy for our reaction [23] and this is in fair agreement with previously established systematics for almost mass-symmetric systems [24]. Therefore for the subsequent statistical model analysis of the high-energy $\gamma$-ray spectra it was assumed that the compound nucleus is formed at the full excitation energy reached in the complete fusion reaction.

\section{STATISTICAL MODEL ANALYSIS}

The experimental high-energy $\gamma$-ray spectra, measured in coincidence with the evaporation residues, result from the gamma emission that occurs at several steps in the decay cascade, i.e., they are emitted not only by the compound nucleus, but by many evaporation daughter nuclei at various temperatures and spins. Therefore one has to note, that the information extracted from such spectra will be averaged over whole fusion-evaporation process. In what follows we refer to the corresponding averaged spectra as effective.

To obtain the effective giant dipole resonance characteristics such as the centroid energy $E_{\mathrm{GDR}}$, the strength $S$, and the width $\Gamma_{\mathrm{GDR}}$, the high-energy $\gamma$-ray spectra were analyzed in the conventional way [3-6], namely with the help of the statistical model. In the present studies, the statistical code GEMINI++ with an option allowing to treat explicitly the GDR emission [25] was employed for the first time for such an analysis.

The GEMINI++ is a Monte Carlo code, producing event-byevent data, which can be sorted out in various distinct ways. It allows to obtain in particular the population matrices of the nuclei created in the evaporation process. These matrices can 

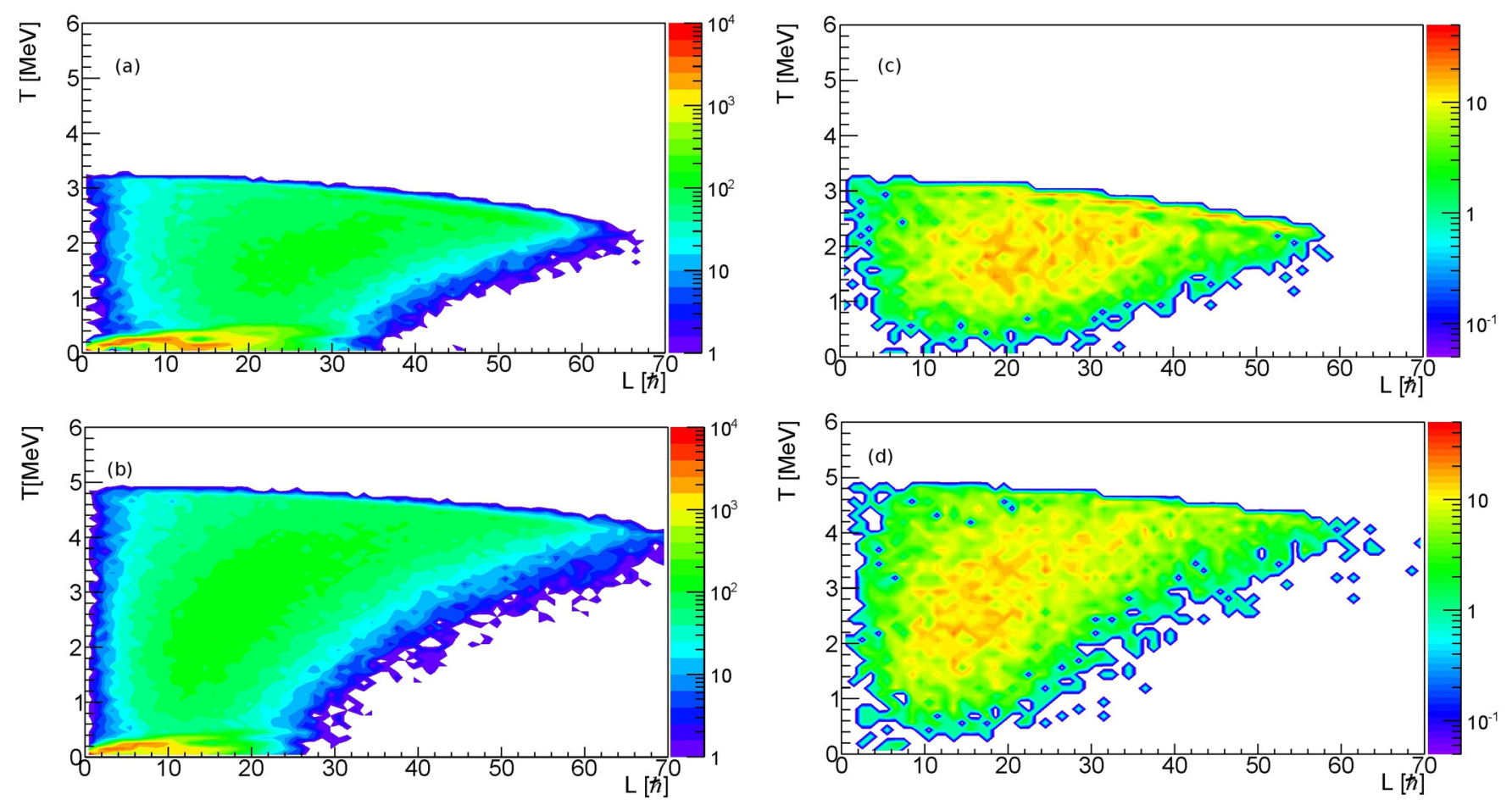

FIG. 8. (Color online) Left panel: The population matrices, calculated using the GEMINI++ code, of all nuclei through which the evaporation process of ${ }^{88}$ Mo proceeds, with an imposed condition that in the evaporation chain the residuum is reached. Two-dimensional spectra show the numbers of such nuclei at any given spin and temperature in a logarithmic scale for the reactions (a) at $300 \mathrm{MeV}$ and (b) at $600 \mathrm{MeV}$. Right panel: Analogous to the left panel, but with an additional condition: Only the nuclei emitting a $\gamma$ ray with $E_{\gamma}>11 \mathrm{MeV}$ (i.e., from the GDR decay) are considered.

be represented as functions of the nuclear spin and temperature (see below) with different conditions superposed (see Fig. 8). When the compound nucleus is formed at very high excitation energies, it de-excites via multiple emissions of neutrons and charged particles. This implies that the average temperature and spin after each evaporation event are much lower then their original values for the compound nucleus (see Table II). One can also notice that the average angular momentum for the compound nucleus is somewhat lower for $600 \mathrm{MeV}$ case than for the $300 \mathrm{MeV}$. This reflects the growing effect of the fission process with increasing excitation energy.

The $\gamma$ decay of the GDR is possible at each step of the evaporation process, i.e., from nuclei characterized by different

TABLE II. Parameters of the ${ }^{48} \mathrm{Ti}+{ }^{40} \mathrm{Ca}$ reaction calculated by GEMINI++ with experimental conditions imposed. Meaning of symbols: $E_{\mathrm{LAB}}^{b}$ : beam energy; $\left\langle T_{\mathrm{CN}}\right\rangle$ : mean $\mathrm{CN}$ temperature; $\left\langle T_{\mathrm{EV}}\right\rangle$ : mean temperature of nuclei in the evaporation process after the emission of GDR $\gamma$ ray; $\left\langle l_{\mathrm{CN}}\right\rangle$ : mean $\mathrm{CN}$ angular momentum; $\left\langle l_{\mathrm{EV}}\right\rangle$ : mean angular momentum of nuclei in the evaporation process after emission of the GDR $\gamma$ ray; $\langle N\rangle$ and $\langle Z\rangle$ : mean $N$ and $Z$ of nuclei in the evaporation process emitting GDR $\gamma$ ray.

\begin{tabular}{lcccccc}
\hline \hline $\begin{array}{l}E_{\mathrm{LAB}}^{b} \\
(\mathrm{MeV})\end{array}$ & $\begin{array}{c}\left\langle T_{\mathrm{CN}}\right\rangle \\
(\mathrm{MeV})\end{array}$ & $\begin{array}{c}\left\langle T_{\mathrm{EV}}\right\rangle \\
(\mathrm{MeV})\end{array}$ & $\begin{array}{c}\left\langle l_{\mathrm{CN}}\right\rangle \\
(\hbar)\end{array}$ & $\begin{array}{c}\left\langle l_{\mathrm{EV}}\right\rangle \\
(\hbar)\end{array}$ & $\langle N\rangle$ & $\langle Z\rangle$ \\
\hline 300 & 3 & 2.0 & 43 & 24 & 45 & 40 \\
600 & 4.5 & 3.1 & 41 & 20 & 44 & 36 \\
\hline \hline
\end{tabular}

temperatures and spins; for illustration see panels (c) and (d) in Fig. 8.

The evaporation process in the GEMINI ++ code is described by the Hauser-Feschbach formalism [26], in which the decay width for the evaporation of an $i$ th particle from the compound nucleus, with an excitation energy of $E^{*}$ and spin $s_{\mathrm{CN}}$, is given by the expression

$$
\begin{aligned}
\Gamma_{i}= & \frac{1}{2 \pi \rho\left(E^{*}, s_{\mathrm{CN}}\right)} \\
& \times \int d \epsilon \sum_{s_{d}=0}^{\infty} \sum_{J=\left|s_{\mathrm{CN}}-s_{d}\right|}^{s_{\mathrm{CN}}+s_{d}} \sum_{\ell=\left|J-s_{i}\right|}^{J+s_{i}} T_{\ell}(\epsilon) \rho\left(E^{*}-B_{i}-\epsilon, s_{d}\right) .
\end{aligned}
$$

Above, $s_{d}$ is the spin of the daughter nucleus, $s_{i}, J$, and $\ell$ are, respectively, the spin, the total momentum, and the orbital momentum of the evaporated particle, $\epsilon$ and $B_{i}$ are kinetic and separation energies, $T_{\ell}$ are transmission coefficients, and $\rho$ and $\rho_{\mathrm{CN}}$ are level densities of the daughter and compound nuclei, respectively. They have been calculated using the expression [21]

$$
\rho(U, s)=\frac{(2 s+1)}{24 \sqrt{2}\left(1+U^{5 / 4} \sigma^{3}\right) \sqrt[4]{a(U, s)}} \exp (2 \sqrt{a(U, s) U}),
$$

where $\sigma=\sqrt{\mathcal{J} T}$, with $\mathcal{J}$ being a moment of inertia of a rigid body with the same density as the nucleus. In this context the 
$T$ is the nuclear temperature defined as

$$
\frac{1}{T}=\frac{d S}{d U} \leftrightarrow S=2 \sqrt{a(U, s) U},
$$

where $S$ stands for the nuclear entropy, and where

$$
U=E^{*}-E_{\mathrm{rot}}(s)+\delta P+\delta W
$$

is the thermal excitation energy, calculated taking into account the pairing $\delta P$ and the shell corrections $\delta W$ to the empirical mass formula. Finally, $E_{\text {rot }}(s)$ denotes the rotational energy of the nucleus, here taken in the form of the rotating liquid drop model energy expression with the rigid-body moment of inertia.

The level density parameter $a(U, s)$ was parametrized after Ref. [21] as

$$
a(U, s)=\tilde{a}(U)\left(1-h\left(U / \eta+s / s_{\eta}\right) \frac{\delta W}{U}\right),
$$

where $\delta W$ is the shell correction to the liquid-drop mass and $\tilde{a}$ is the smoothed level-density parameter (see below). The separation energies $B_{i}$, nuclear masses, and shell and pairing corrections were taken from Ref. [27]. The function specifying the rate of fadeout is $h(x)=\tanh x$, with fadeout parameters $\eta=18.52$ [28] and $s_{\eta}=50 \hbar$ [21].

The smoothed level density parametrization depends on the nuclear excitation energy according to the following phenomenological relation:

$$
\tilde{a}(U)=\frac{A}{k_{\infty}-\left(k_{\infty}-k_{0}\right) \exp \left(-\frac{\kappa}{k_{\infty}-k_{0}} \frac{U}{A}\right)},
$$

including the set of parameters $k_{0}=7.3 \mathrm{MeV}, k_{\infty}=12 \mathrm{MeV}$, and $\kappa=0.00517 \exp (0.0345 A)$, which were taken from [21]. However, for the mass region below $\mathrm{A}=100$ the dependence on nuclear excitation energy is small [21]. In the case of the experimental data of interest here, i.e. for masses of the studied nuclei and excitation energy $U$ up to $260 \mathrm{MeV}$, the values of $\tilde{a}$ lie in the range from $\frac{A}{7.3}$ to $\frac{A}{7.6} \mathrm{MeV}^{-1}$.

The effective GDR strength function was extracted from the data in the following way. First, we introduce the trial GDR strength function $L\left(E_{\gamma}\right)$ which is defined as the sum of three Lorentz-form components as follows:

$$
L\left(E_{\gamma}\right)=\sum_{k=1}^{3} \frac{S_{k} E_{\gamma}^{2} \Gamma_{k}}{\left(E_{\gamma}^{2}-E_{k}^{2}\right)^{2}+E_{\gamma}^{2} \Gamma_{k}^{2}},
$$

with adjustable parameters $E_{k}, S_{k}$, and $\Gamma_{k}$, for $k=1,2,3$. This expression was inserted into the GEMINI++ code, and the whole evaporation process was simulated in the event-by-event mode.

In the calculations the compound nucleus angular momentum distribution was based on the Bass prescription $[15,16]$. The charged-particle and $\gamma$-ray events generated by the statistical code were subsequently sorted using the same conditions as in the experiment (i.e., by selecting only such events in which residual nuclei were emitted to the solid angles covered by the detectors). The first iteration of the model $\gamma$-ray spectrum was obtained using certain starting-value parameters in the energy interval from 8 to $24 \mathrm{MeV}$, compared with the experimental one, and the corresponding $\chi^{2}$ value was calculated. Then,
TABLE III. The best fit GDR parameters: centroid $E_{k}$, width $\Gamma_{k}$, and strength $S_{k}$ adjusted to the experimental data on the decay of ${ }^{88} \mathrm{Mo}$ formed in the reaction ${ }^{48} \mathrm{Ti}+{ }^{40} \mathrm{Ca}$ at beam energies of 300 and $600 \mathrm{MeV}$.

\begin{tabular}{lrrr}
\hline \hline$E_{\mathrm{LAB}}^{b}$ & $E_{k}(\mathrm{MeV})$ & $\Gamma_{k}(\mathrm{MeV})$ & \multicolumn{1}{c}{$S_{k}$} \\
\hline $300 \mathrm{MeV}$ & $10.2 \pm 1.3$ & $4.5 \pm 1.1$ & $0.05 \pm 0.02$ \\
& $14.7 \pm 0.4$ & $8.0 \pm 0.3$ & $0.92 \pm 0.03$ \\
& $20.2 \pm 0.4$ & $11.0 \pm 1.3$ & $0.22 \pm 0.03$ \\
$600 \mathrm{MeV}$ & $9.8 \pm 0.5$ & $4.4 \pm 1.4$ & $0.09 \pm 0.03$ \\
& $14.7 \pm 0.2$ & $7 \pm 0.8$ & $0.52 \pm 0.03$ \\
& $20.2 \pm 0.5$ & $9 \pm 1.9$ & $0.19 \pm 0.04$ \\
\hline \hline
\end{tabular}

a new trial GDR function was inserted and new spectrum calculated, and again compared with the experimental one. In such an iterative process, adjusting the values of the GDR parameters in order to match the experimental spectra in the GDR region, the best $\chi^{2}$ fit was obtained.

The best-fit values are reported in Table III for the two bombarding energies studied here. The high-energy $\gamma$-ray spectra, the experimental one and the one fitted with the statistical model, normalized at $8 \mathrm{MeV}$, are presented in Fig. 9, showing an overall good agreement.

To illustrate the yield of the GDR and the quality of the GEMINI++ fit on a linear scale, the experimental spectra were converted to the GDR strength functions, $Y_{\exp }\left(E_{\gamma}\right)$, using the method described in Ref. [29], i.e., by dividing the experimental spectrum by the calculated one, and multiplying by the best fit GDR strength function $L\left(E_{\gamma}\right)$. The corresponding results are illustrated in Fig. 10, where also the best-fit GDR strength function, $L\left(E_{\gamma}\right)$ with three components, is shown.

The effective values of the $\Gamma_{\mathrm{GDR}}$ widths obtained (for both experimental data sets) as the FWHM best fit of the single Lorentz function, as well as the experimental strength

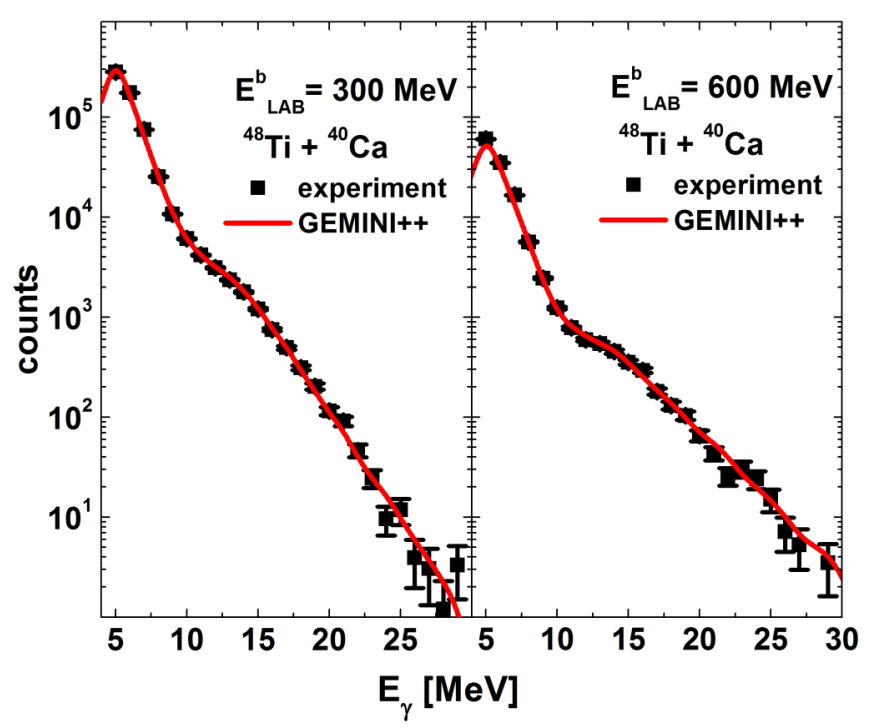

FIG. 9. (Color online) A comparison of the $\gamma$-ray spectra from the ${ }^{48} \mathrm{Ti}+{ }^{40} \mathrm{Ca}$ reaction, at the beam energies of 300 and $600 \mathrm{MeV}$, with the results of the GEMINI++ fit (see text). 


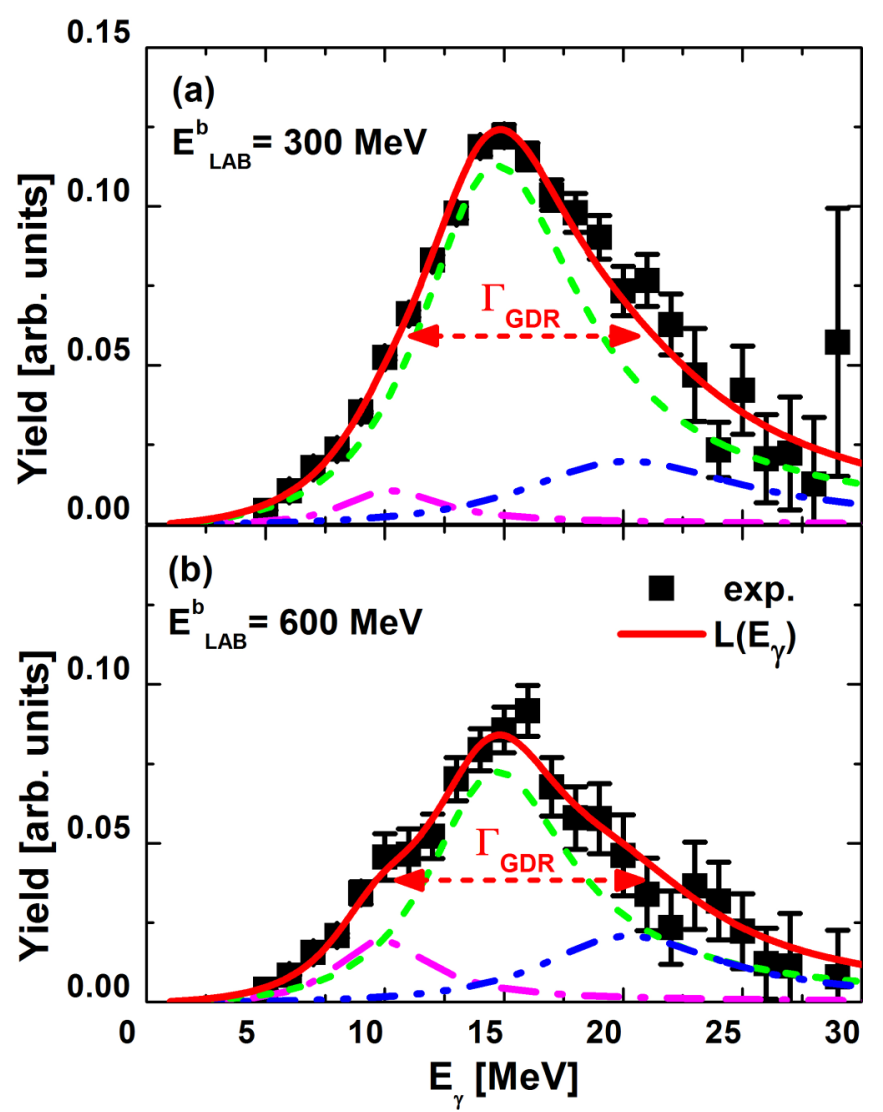

FIG. 10. (Color online) The experimental, $Y_{\exp }\left(E_{\gamma}\right)$, and fitted, $L\left(E_{\gamma}\right)$, GDR strength functions for (a) 300 and (b) $600 \mathrm{MeV}$ beam energies.

functions of Fig. 10, were used in the following discussions and comparisons with theoretical models. The deduced values of the effective GDR energies (centroids), GDR widths (FWHM), and the total GDR strength functions (being sum of strength functions for individual GDR components) for the data at the beam energies 300 and $600 \mathrm{MeV}$ are given in Table IV. The extracted values of the $S_{\mathrm{GDR}}$ are 1.19(5) and 0.80(5) for 300 and $600 \mathrm{MeV}$, respectively. The noticeable smaller value for $600 \mathrm{MeV}$ (what corresponds to $261 \mathrm{MeV}$ excitation energy of the ${ }^{88} \mathrm{Mo} \mathrm{CN}$ ) might support the hypothesis and recent findings of the onset of the GDR strength quenching at high excitation energy (see [30-32] and references therein).

The average temperature of nuclei, in which the GDR is excited and gives the contributions to the measured $\gamma$ spectra, is lower than the temperature of the compound nucleus. The values of the nuclear temperature corresponding to the GDR emitting system are estimated (see Ref. [21]) at each decay

TABLE IV. GDR centroid energies $E_{\mathrm{GDR}}$, effective widths $\Gamma_{\mathrm{GDR}}$, and total strengths $S_{\mathrm{GDR}}$ for the decay of ${ }^{88} \mathrm{Mo}$.

\begin{tabular}{lccc}
\hline \hline$E_{\mathrm{LAB}}^{b}$ & $E_{\mathrm{GDR}}(\mathrm{MeV})$ & $\Gamma_{\mathrm{GDR}}(\mathrm{MeV})$ & $S_{\mathrm{GDR}}$ \\
\hline $300 \mathrm{MeV}$ & $14.9 \pm 0.2$ & $9.9 \pm 0.7$ & $1.19(5)$ \\
$600 \mathrm{MeV}$ & $14.7 \pm 0.2$ & $10.3 \pm 0.9$ & $0.80(5)$ \\
\hline \hline
\end{tabular}
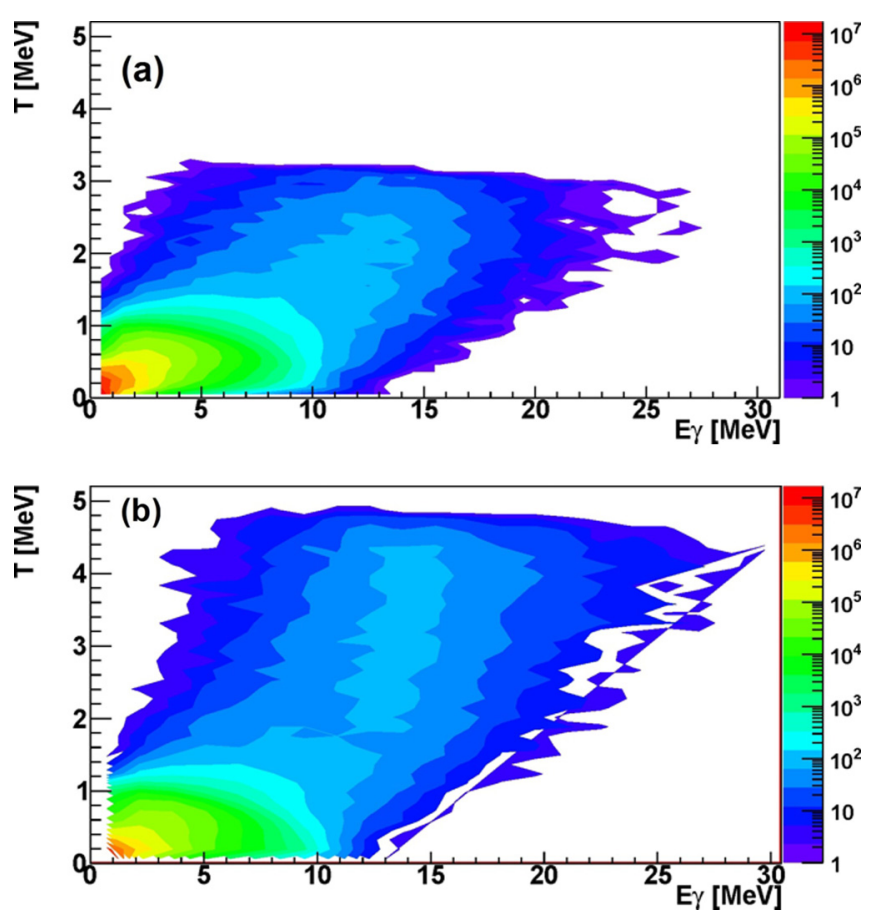

FIG. 11. (Color online) Distribution of the nuclei in the evaporation process as functions of the nuclear temperature reached after the $\gamma$ decay and the emitted $\gamma$-ray energy; calculated with the code GEMINI++ for the data at the beam energies of (a) $300 \mathrm{MeV}$ and (b) $600 \mathrm{MeV}$.

step using the expression

$$
T=\left[\left(U-E_{\mathrm{GDR}}\right) / a(U, s)\right]^{\frac{1}{2}},
$$

where $E_{\mathrm{GDR}}$ is the energy of the emitted $\gamma$ ray.

The GEMINI++ code allows us to examine the decay process of compound nuclei at each decay step, and to obtain several quantities useful for the experimental data analysis. For example the events produced by the GEMINI++ code can be sorted out into another population matrix, as a function of the $\gamma$-ray energy and temperature of the nucleus after $\gamma$ emission. Such population distributions obtained for both data sets are shown in Fig. 11 and can be an important help in estimating the average temperature and the range of temperatures of nuclei, on which the GDR is built.

Examining the results in Fig. 11 brings us to the conclusion that the most significant part of the 2D spectra corresponds to the temperatures below $1.2 \mathrm{MeV}$ and thus that the spectrum in question originates mainly from the statistical $\gamma$ rays emitted below the particle threshold. The contribution from this radiation is important up to $11 \mathrm{MeV}$, and above this energy we have the $\gamma$ rays exclusively from the decay of the GDR. Therefore to estimate the distributions of the temperatures involved in the GDR decay only the events with $E_{\gamma}>11 \mathrm{MeV}$ were considered.

The temperature distributions attributed to the GDR emission, namely corresponding to the $\gamma$-ray energies $E_{\gamma}>$ $11 \mathrm{MeV}$, are plotted in Fig. 12. As the effective temperature, characteristic of the evaporation process at given bombarding energy, the average temperature of the distribution is 


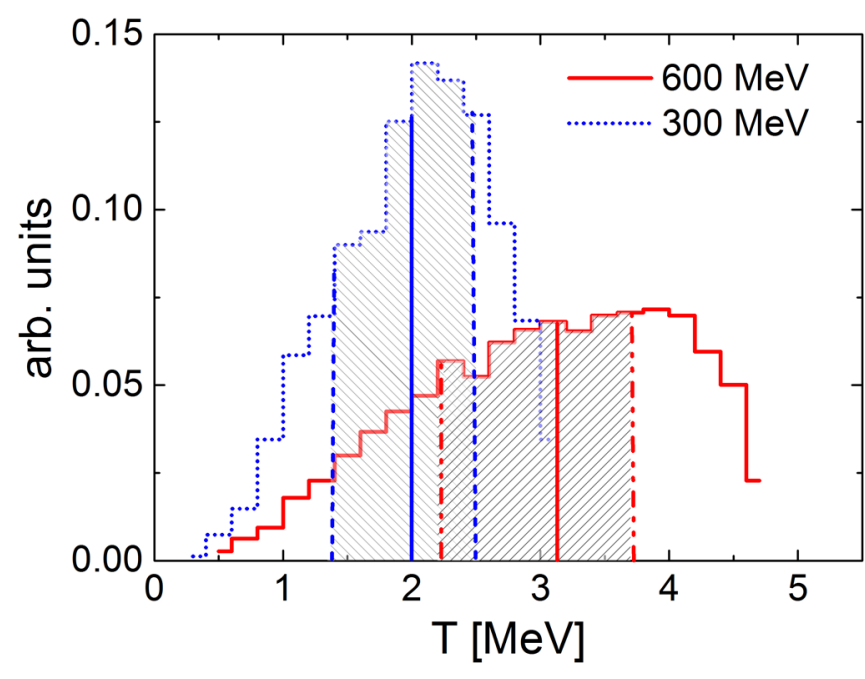

FIG. 12. (Color online) Temperature distributions of the number of nuclei after the GDR emission. The calculations were performed using the GEMINI++ code with experimental conditions imposed (see the text for details). The blue (dotted) and red (solid) histograms represent the data at the beam energies of 300 and $600 \mathrm{MeV}$, respectively. The vertical solid lines indicate the average values of the distributions. The dashed and dot-dashed vertical lines show corresponding quartiles used for estimation of temperature uncertainties.
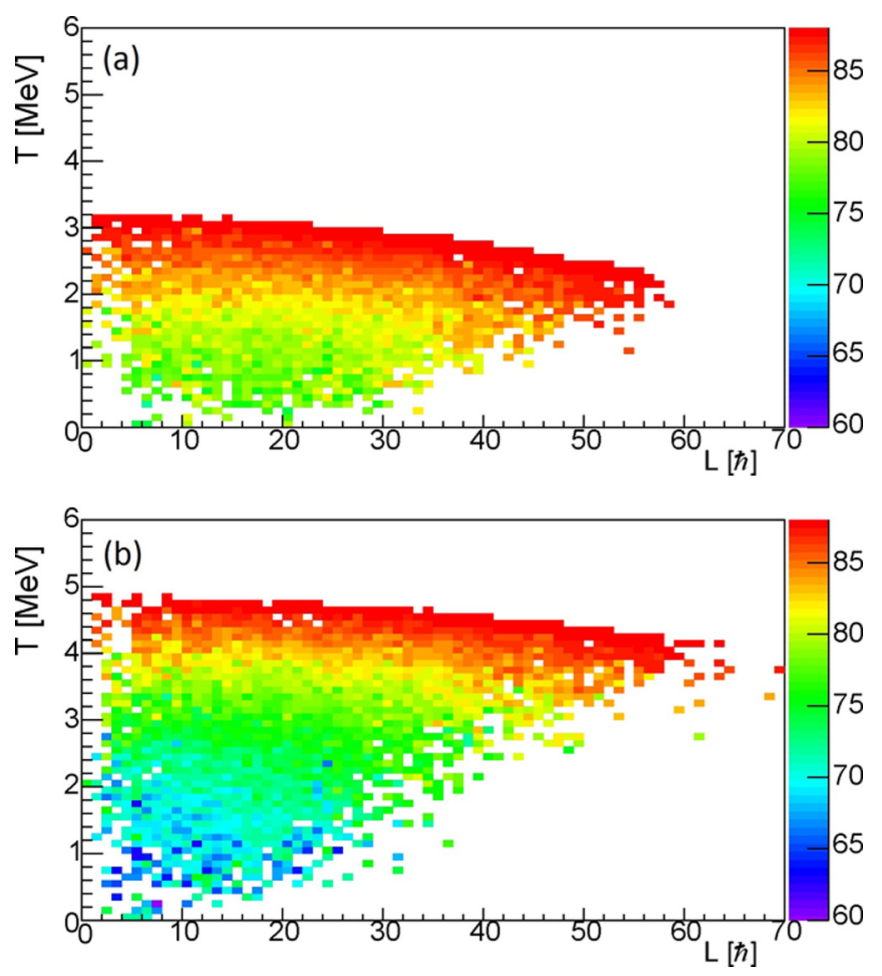

FIG. 13. (Color online) Average mass $\langle A\rangle$ (z axis) of a nucleus emitting GDR $\gamma$ rays during the decay of the ${ }^{88}$ Mo compound nucleus produced at (a) $300 \mathrm{MeV}$ and (b) $600 \mathrm{MeV}$ beam energies as functions of temperature and spin. Calculations were performed using the statistical code GEMINI ++ . taken, with the error estimated as lower and upper quartiles. In this way the obtained effective temperatures at the beam energies of 300 and $600 \mathrm{MeV}$ are $\left\langle T_{\mathrm{EV}}\right\rangle=2.0_{-0.6}^{+0.5}$ and $3.1_{-0.9}^{+0.6}$ $\mathrm{MeV}$, respectively. These values were subsequently used for comparisons with models and other experimental results.

Another useful piece of information that can be extracted from the data produced by the GEMINI ++ code can be obtained in the form of the population matrices of the average nuclear mass $\langle A\rangle$ at a given temperature and spin after the decay through the GDR $\gamma$ emission. Those matrices are obtained by employing the experimental conditions; in the present case the coincidence between evaporation residue and the GDR $\gamma$ emission. Examples are presented in Fig. 13.

The mean angular momentum for the decaying nuclei emitting GDR $\gamma$ rays obtained using the GEMINI++ simulations was $24 \hbar$ and $20 \hbar$ for the beam energies of 300 and $600 \mathrm{MeV}$, respectively (see Table II). These values are much lower, as compared to the average angular momentum of the compound nuclei of ${ }^{88} \mathrm{Mo}$, estimated to be $43 \hbar$ for the data at a beam energy of $300 \mathrm{MeV}$ and $41 \hbar$ for $600 \mathrm{MeV}$. These differences can be attributed to considerable amounts of angular momentum removed by the light particles during the evaporation process.

\section{MODELING THE EFFECTIVE GDR STRENGTH FUNCTION}

In order to interpret the experimental information on the GDR strength functions and on the effective GDR widths, theoretical approaches using two distinct techniques of modeling the giant resonance were employed. The first one is based on the recent version of the liquid drop model, LSD, see Refs. $[33,34]$, supplemented with the thermal shape fluctuation method (TFM) in the realization analogous to the one discussed in [6] and [35], whereas the other one is the phonon damping model (PDM) of [36].

\section{A. Lublin-Strasbourg drop model and thermal shape fluctuations}

The properties of the nuclear GDR behavior at high temperatures and spins can be simulated using the nuclear mean-field theory in its various realizations together with the TFM approach just mentioned. Here we follow closely [6,35], see also [37,38], certain applications of this approach can be found in the articles [39-43].

Within the nuclear mean-field theory one controls directly the nuclear-shape degrees of freedom either through the expected values of the multipole moments, as in the case of the self-consistent theories, or more directly through the imposed shapes of the nuclear surface, as in the case of the macroscopicmicroscopic methods. Of central interest here will be the density of probability to find a given nucleus at any given shape, for a physicist-defined temperature $T$ and angular momentum $I$. It can be estimated using the standard expression from the nuclear thermodynamics:

$$
P(\alpha ; I, T) \propto \exp \{-F(\alpha ; I, T) / k T\}
$$


where $\alpha \equiv\left\{\alpha_{\lambda \mu}\right\}$ denotes the ensemble of all the deformation parameters used within the mean-field model; for instance $\lambda=2$ and $\mu=0$ correspond to axial, and $\lambda=2$ and $\mu=2$ to nonaxial quadrupole deformations. Above, $F(\alpha ; I, T)$ denotes the nuclear free energy, whereas $T$ is the nuclear temperature and $k$ the Boltzmann constant.

The approach based on the use of the shape probability distribution of Eq. (9) is referred to as thermal shape fluctuation method (TFM). The name stresses the fact that the varying temperature causes an evolution of the the deformation space areas in which the system resides preferentially.

The nuclear free energy is defined as usual by

$$
F(\alpha ; I, T)=E(\alpha ; I)-T S,
$$

where $E(\alpha ; I)$ denotes the nuclear energy which in our case will be obtained within the mean-field theory and $S$ the entropy of the nucleus (see below). In the present context, the total nuclear energy can be conveniently calculated using the macroscopic-microscopic method of Strutinsky. Since, however, we are interested in this article in the nuclear states at the relatively high temperatures of the order of 1 to $3 \mathrm{MeV}$, we will ignore as a matter of approximation the nuclear shell and pairing effects, which allows limiting the expression of the total nuclear energy to the macroscopic, liquid-drop-model part only.

In the present case we will employ the Lublin-Strasbourg drop (LSD) realization of the latter, and it may be instructive at this point to present some comments about this particular choice; see Refs. [33,34] for details. The parameters of the LSD model have been adjusted to the experimental data on the nuclear masses. Introducing the surface curvature terms, which has been an essential new element of the LSD approach, allowed us to reduce the discrepancies between the model and the experiment in terms of the fission barriers considerably while using the same experimental information about the masses (no extra fits of the model parameters to the fission barriers have been allowed). This result demonstrates the intrinsic correctness of the physics assumptions underlying the surface curvature mechanism within the LSD model, as well as that the dependence of the nuclear energy on the deformation is well controlled within this approach. Since the shape effects play the first-rank role in the present study, employing the LSD model seems to be the right choice to profit from a good empirical correspondence between the nuclear energies and shapes.

The LSD energy has been calculated in a five dimensional deformation space $\left(\alpha_{20}, \alpha_{22}, \alpha_{40}, \alpha_{60}, \alpha_{80}\right)$ and projected onto the quadrupole, the so-called $(\beta, \gamma)$ plane with $\alpha_{20}=\beta \cos \gamma$ and $\alpha_{22}=(\beta / \sqrt{2}) \sin \gamma$. For each given spin of interest and every $(\beta, \gamma)$ point [equivalently $\left(\alpha_{20}-\alpha_{22}\right)$ point] the total energy, including its rotational part, Eq. (11), was minimized over the remaining three deformations. The nuclear rotation was treated using the classical approximation and in this way the nuclear energy expression $E(\alpha, I)$ in Eq. (10) was taken in its macroscopic-energy form depending on the nuclear deformation and spin as follows:

$$
E(\alpha, I) \rightarrow E_{\text {macro }}(\alpha ; I)=E_{\mathrm{LSD}}(\alpha)+\frac{\hbar^{2}}{2 \mathcal{J}(\alpha)} I(I+1)
$$

Above, $\mathcal{J}(\alpha)$ denotes the deformation-dependent classical moment of inertia.
$\mathrm{I}=40 \mathrm{~h}, \Gamma_{0}=5 \mathrm{MeV}$ (a)

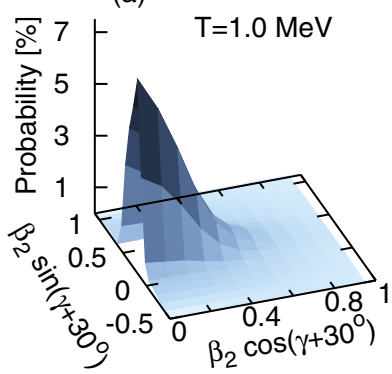

(c)

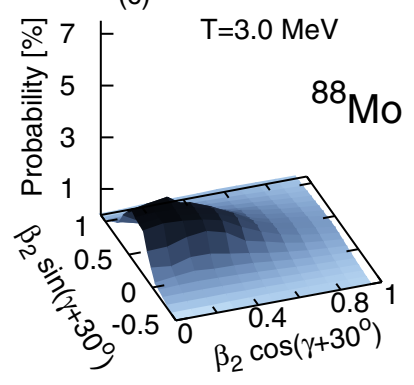

(b)

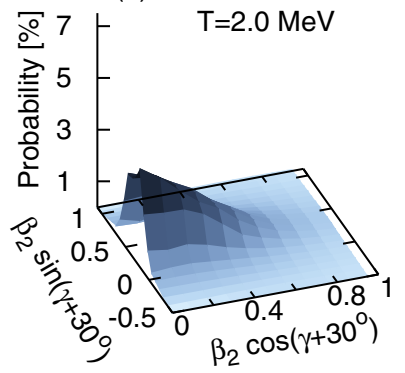

(d)

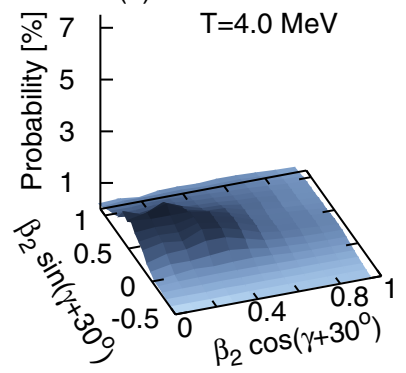

FIG. 14. (Color online) The nuclear-shape probability distributions for ${ }^{88} \mathrm{Mo}$ at temperatures $T=1,2,3$, and $4 \mathrm{MeV}$ and spin $40 \hbar$ in panels (a), (b), (c), and (d), respectively. The energy minimization is performed over $\alpha_{40}, \alpha_{60}$, and $\alpha_{80}$ and the results are projected onto the $(\beta, \gamma)$ plane.

In order to obtain the entropy, which is a necessary element in the free-energy definition, we use the fact that we have at our disposal the nuclear single-particle energies, here obtained with the help of the deformed Woods-Saxon Hamiltonian with the so-called universal parameters of Ref. [44]. Using this information the entropy has been calculated from Eq. (4) of Ref. [35], in which also the references to the earlier publications are given.

The resulting probability distributions for ${ }^{88} \mathrm{Mo}$, with the angular momentum of $40 \hbar$, at various temperatures selected as 1, 2, 3, and $4 \mathrm{MeV}$ are presented in Fig. 14, as an example. As it can be seen from the figure, increasing temperature implies a flattening of the probability distributions over the $(\beta, \gamma)$ plane.

These probability distributions are used for applying the thermal shape fluctuation method: the calculated GDR strength functions for each set of deformation parameters $\beta$ and $\gamma$ are averaged over the $(\beta, \gamma)$ plane using the probabilities as weighting factors. The deformation dependence of the GDR strength function can be conveniently modeled using the algorithm proposed by Nergaard [45]. Here we follow Eq. (15) of Ref. [35] and define the quantity $\mathcal{L}\left(E_{\gamma},\left\{E_{k}\right\} ; \beta, \gamma ; I, T\right)$, which, apart from a slight modification in the notation, has the interpretation of the GDR strength function built on the nucleus with the deformation parameters $(\beta, \gamma)$, spin $I$, and temperature $T$. It can be represented with the help of five Lorentz components ${ }^{1}$ centered at the energies $E_{k}(\mathrm{k}=1, \ldots, 5)$

\footnotetext{
${ }^{1}$ This is so because one can show that at sufficiently high spins, for the nucleus turning about its, say, $y$ axis, there is only one giant-
} 
the latter depending both on the deformation and on the spin. The symbol $\left\{E_{k}\right\}$ means ensemble of all the five Lorentz components so that $\mathcal{L}$ depends in general on all the five of them as well.

In the present article, the widths of the considered components were parametrized as follows:

$$
\Gamma_{k}=\Gamma_{0}\left(E_{k} / E_{\mathrm{GDR}}\right)^{\delta},
$$

where $\Gamma_{0}$ is the GDR width at $T=0$ and the GDR energy

$$
E_{\mathrm{GDR}}=18 A^{-1 / 3}+25 A^{-1 / 6},
$$

from Ref. [46], whereas $\delta$ is taken as 1.9, from [47].

In this method of modeling the GDR strength function, an essential parameter is the intrinsic width $\Gamma_{0}$. Usually it is assumed that $\Gamma_{0}$ does not depend on temperature and is equal to the GDR width appropriate for the ground state of any given nucleus. In what follows, $\Gamma_{0}$ was chosen to be $5 \mathrm{MeV}$, because this value is close to the experimentally known data in this region of nuclei.

After taking the thermal shape fluctuation into account at any given temperature and spin, one obtains a GDR strength function $L_{\mathrm{TFM}}$ for the whole shape ensemble. It is defined as the deformation probability-weighted integral of the profiles $\mathcal{L}$ for each deformation set:

$$
\begin{aligned}
& L_{\mathrm{TFM}}\left(E_{\gamma} ; I, T\right) \\
& \quad=\int P(\beta, \gamma ; I, T) \mathcal{L}\left(E_{\gamma},\left\{E_{k}\right\} ; \beta, \gamma ; I, T\right) \beta d \beta d \gamma .
\end{aligned}
$$

The new quantity on the left-hand side is an effective (deformation-averaged) GDR strength function.

Figure 15 shows the strength functions for each temperature of Fig. 14, and for the angular momenta of $I=24 \hbar, 40 \hbar$, and $50 \hbar$. The increase in temperature produces a broadening of the GDR strength function, since various nuclear shapes with larger and larger deformations become probable. The splitting of the GDR strength function into two or more components can be caused by the presence and variation of the most probable deformations with increasing temperature and/or spin. A typical shape variation with spin according to which the spherical shape (at null angular momentum) evolves to an axial-oblate one at moderate spins, next to triaxial and to prolate ones at still increasing angular momentum (see Refs. [39,40] for illustrations), can be accompanied by and/or compete with analogous shape changes as a function of increasing temperature.

The assumption that the intrinsic width $\Gamma_{0}$ is the same as for the ground state even at very high temperatures might not be correct, as pointed out in Refs. [12,48]. Indeed, the lifetime of the compound nucleus, as well as the lifetimes of nuclei during the evaporation process may play an important role at

resonance frequency associated with the $y$-axis oscillation/phonon, whereas there are two distinct giant resonance frequencies associated with the $x$ and $z$ axes [45]. For not very high spins (not sufficiently high rotation) the splitting just mentioned may be considered negligible and the system of five Lorentzians can be reduced, as an approximation, to the system of three Lorentzians only.
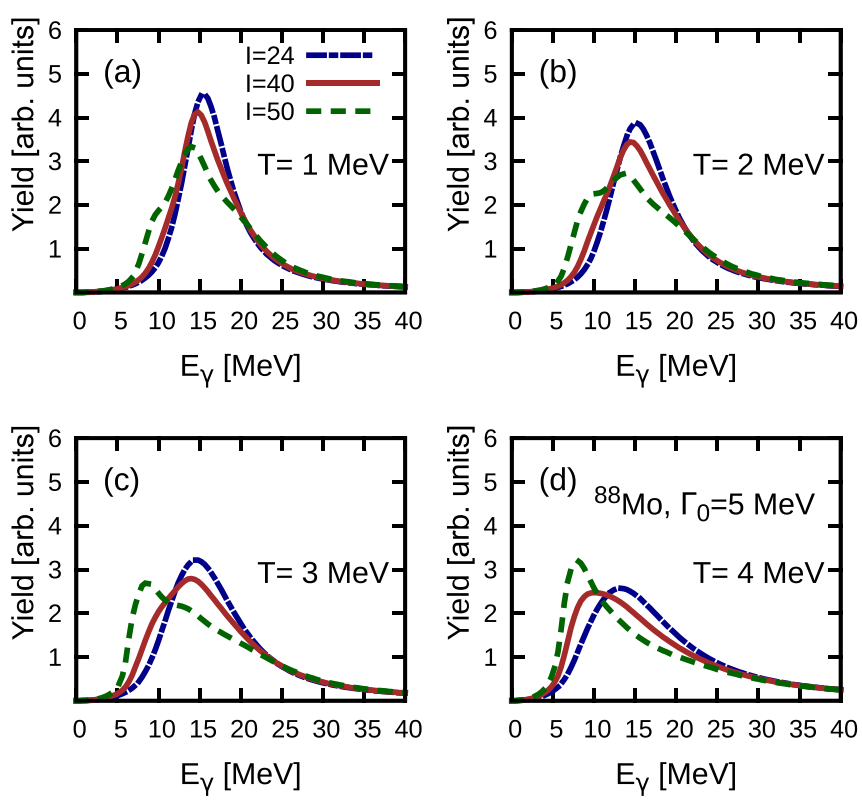

FIG. 15. (Color online) The calculated GDR strength functions at various temperatures and spins for the ${ }^{88}$ Mo nucleus, averaged using the shape probability distributions shown in Fig. 14, with $\Gamma_{0}=$ $5 \mathrm{MeV}$.

very high temperatures, and this mechanism generally needs to be taken into account.

In the present article, the evaporation widths $\Gamma_{\mathrm{EV}}$, corresponding to the lifetimes of compound nucleus and of nuclei produced in the evaporation process, were calculated by the GEMINI++ code at each decay step and they are shown in Fig. 16, top.

Evaporation widths calculated for different temperatures were fitted with the exponential functions of the form $C \exp (T)$, where $C=0.046$ is a fitted parameter. The value $\Gamma_{\mathrm{EV}}(T)$ obtained as a result of the fit represented by the line in Fig. 16, top, was included in the parametrization of the intrinsic GDR width $\Gamma_{0}(T)$ by adding it to the value of $\Gamma_{0}(T=0)=5 \mathrm{MeV}$, which is the GDR width at $T_{\mathrm{GDR}}=0$. The obtained parametrization of the intrinsic GDR width, $\Gamma_{0}(T)=\Gamma_{0}(T=0)+2 \Gamma_{\mathrm{EV}}$, is presented in Fig. 16, bottom. This definition was used in the thermal shape fluctuation approach for calculations of the form of the GDR strength functions [Eq. (14)].

The GDR strength functions modeled with the help of $L_{\mathrm{TFM}}$, see Eq. (14), were calculated for each temperature and spin in the range spanned by the evaporation process as shown in Fig. 8. Since the GDR strength function depends on the nuclear mass [Eq. (13)], the average mass corresponding to any given temperature and spin was calculated from the results in Fig. 13. In order to compare them to those experimentally extracted and shown in Fig. 10, an effective GDR strength function was computed by convoluting $L_{\mathrm{TFM}}$ functions at each temperature and spin with the population matrices $P_{p}(I, T)$ from GEMINI++ presented in Figs. 8(c) and 8(d):

$$
L_{\text {effective }}^{\mathrm{TFM}}\left(E_{\gamma}\right)=\sum_{I, T} P_{p}(I, T) L_{\mathrm{TFM}}\left(E_{\gamma} ; I, T\right) .
$$




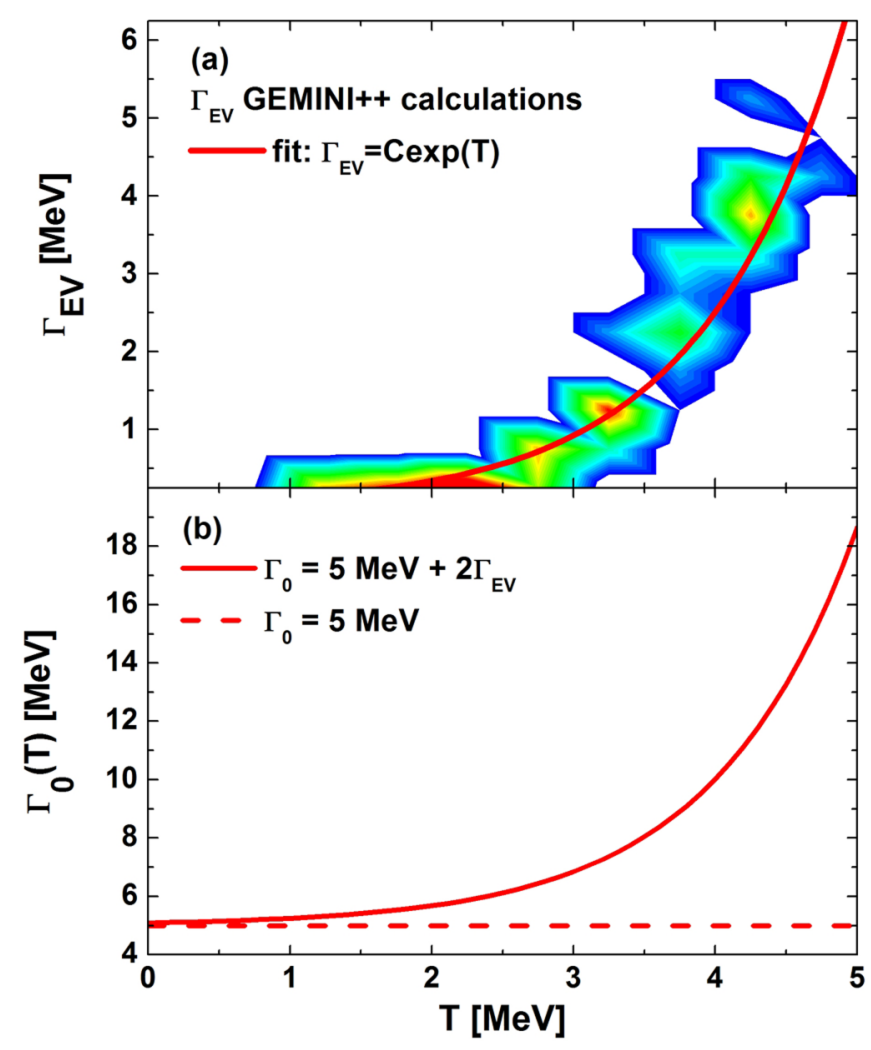

FIG. 16. (Color online) (a) The evaporation widths $\Gamma_{\mathrm{EV}}$ calculated using the GEMINI++ code at various temperatures. (b) Intrinsic width of the GDR: a constant $\Gamma_{0}(T)=\Gamma_{0}(T=0)=5 \mathrm{MeV}$ (dashed line) and one dependent on the evaporation width $\Gamma_{\mathrm{EV}}$, in the form of $\Gamma_{0}(T)=5 \mathrm{MeV}+2 \Gamma_{\mathrm{EV}}$ (solid line $)$.

Such an effective GDR strength functions, taking into account both the thermal shape fluctuations at each given spin and temperature, as well as the spin and temperature distributions implied by the fusion-evaporation process and by the experimental conditions, have been used to compare with the experimental ones; see Sec. V.

\section{B. Phonon damping model}

Another model used in this article is the phonon damping model (PDM) [36,49-51] which represents the GDR width $\Gamma(T)$ at a given temperature $T$ as a sum

$$
\Gamma(T)=\Gamma_{Q}(T)+\Gamma_{T}(T)
$$

of the quantal width $\Gamma_{Q}(T)$ and thermal width $\Gamma_{T}(T)$. The quantal width originates from the coupling of the GDR phonon to the particle-hole ( $\mathrm{p}-\mathrm{h}$ ) configurations, whereas the thermal width arises due to the coupling of the GDR phonon to the particle-particle (p-p), and hole-hole (h-h) configurations.

The matrix elements of these couplings are proportional to the differences $\left(f_{s^{\prime}}-f_{s}\right)$ where $f_{s}$ is the single-particle level occupation number and the shorthand notation $\left(s, s^{\prime}\right)$ refers to $\left(s, s^{\prime}\right)=p h, p p^{\prime}, h h^{\prime}$. The single-particle occupation number $f_{s}$ at finite temperature $T$ is described by the Fermi-Dirac distribution

$$
f_{s}=1 /\left\{\exp \left[\left(\epsilon_{s}-\lambda\right) / T\right]+1\right\},
$$

with the single-particle energy $\epsilon_{s}$ and the temperaturedependent Fermi energy (chemical potential) $\lambda$. Therefore the quantal width does not vanish even at $T=0$, since then $f_{h}=1$ and $f_{p}=0$. It is responsible for the spreading width of the GDR built on the ground state, whereas the thermal width vanishes in this case.

At non zero temperatures, the thermal width arises due to coupling to (p-p) and (h-h) configurations. These configurations appear owing to the distortion of the Fermi surface at finite temperature, which leads to nonzero differences $f_{p^{\prime}}-f_{p}$ and $f_{h^{\prime}}-f_{h}$. As $T$ increases, the differences $f_{h}-f_{p}$ decrease resulting in a slight decrease in the quantal width. On the other hand, the differences $f_{p^{\prime}}-f_{p}$ and $f_{h^{\prime}}-f_{h}$ increase sharply with $T$ at low and moderate $T$ to reach a plateau at high $T$, then they decrease as $T$ increases further. This leads to a resulting total width,that increases when the temperature increases up to $T \sim 3 \mathrm{MeV}$ and reaches a plateau at around $T>3-4 \mathrm{MeV}$ in medium and heavy nuclei. This is how the PDM describes the increase in the GDR width at low and moderate temperatures and its saturation at high temperatures [36].

In the open-shell nuclei, the increase in the total width at low temperature is compensated by including temperaturedependent (thermal) pairing in the PDM. Because of thermal fluctuations in finite nuclei, the pairing gap does not collapse at the critical temperature of the superfluid-to-normal phase transition, as is the case in infinite systems, but decreases monotonically with increasing $T$, turning the smooth Fermi surface due to pairing at $T=0$ toward the Heaviside step function, hence reducing the effect of the coupling to (p-p) and (h-h) configurations. This compensation at low $T$ is the reason why the GDR width remains insensitive to temperature at $T \leqslant 1 \mathrm{MeV}$ in open-shell heavy nuclei. At $T>1 \mathrm{MeV}$, the effect of thermal pairing becomes small or negligible so that the GDR width starts to increase because of the increase in the thermal width [49].

A similar mechanism holds for the GDR at finite temperature and angular momentum. To describe the noncollective rotation of a spherical nucleus, the $z$ projection $M$ of the total angular momentum $I$ is added to the PDM Hamiltonian as $-\gamma \hat{M}$, where $\gamma$ is the Lagrangian multiplier, which is sometimes interpreted as the angular velocity or rotation frequency in analogy to classical rotation. This rotation frequency $\gamma$ and the chemical potential $\lambda$ are defined from the equations for total angular momentum $M$ (equal to $I$ for spherical nuclei) and particle number, respectively.

The differences of the occupations numbers in the quantal and thermal widths now become $f_{k^{\prime}}^{ \pm}-f_{k}^{ \pm}$where

$$
f_{k}^{ \pm}=1 /\left[\exp \left(\beta E_{k}^{\mp}\right)+1\right]
$$

and

$$
E_{k}^{\mp}=\epsilon_{k}-\lambda \mp \gamma m_{k}
$$

with $\left(k, k^{\prime}\right)=p h, p p^{\prime}, h h^{\prime}$, and $m_{k}$ are the single-particle positive spin projections.

With the smoothing of the Dirac $\delta$ function by using the Breit-Wigner distribution, the final form of the damping 
half-width $\gamma_{q}\left(E_{\gamma}\right)$ is given as (see Eq. (8) of Ref. [51])

$$
\begin{aligned}
\gamma_{q}\left(E_{\gamma}\right)= & \varepsilon \sum_{k k^{\prime}}\left[\mathcal{F}_{k k^{\prime}}^{(q)}\right]^{2}\left[\frac{f_{k^{\prime}}^{+}-f_{k}^{+}}{\left(E_{\gamma}-E_{k}^{-}+E_{k^{\prime}}^{-}\right)^{2}+\varepsilon^{2}}\right. \\
& \left.+\frac{f_{k^{\prime}}^{-}-f_{k}^{-}}{\left(E_{\gamma}-E_{k}^{+}+E_{k^{\prime}}^{+}\right)^{2}+\varepsilon^{2}}\right] .
\end{aligned}
$$

The GDR width at given temperature and spin is then defined as

$$
\Gamma_{\mathrm{GDR}}^{\mathrm{PDM}}(I, T)=2 \gamma_{q}\left(E_{\gamma}=E_{\mathrm{GDR}}\right),
$$

where $E_{\mathrm{GDR}}$ is the GDR energy. The matrix elements of the GDR phonon coupling are parametrized as $\mathcal{F}_{p h}^{(q)}=\mathcal{F}_{1}$ and $\mathcal{F}_{p p^{\prime}}^{(q)}=\mathcal{F}_{h h^{\prime}}^{(q)}=\mathcal{F}_{2}$ (See Ref. [51] for the details of parameter choice.) A value $\varepsilon=0.5 \mathrm{MeV}$ for the smoothing parameter in Eq. (20) is used in the numerical calculations.

The Lorentzian-like strength function of the GDR for a given temperature and spin is calculated as (see, e.g., Eq. (11) of Ref. [51])

$$
L^{\mathrm{PDM}}\left(E_{\gamma} ; I, T\right)=\frac{E_{\gamma}}{E_{\mathrm{GDR}}}\left[S\left(E_{\gamma}, E_{\mathrm{GDR}}\right)-S\left(E_{\gamma},-E_{\mathrm{GDR}}\right)\right],
$$

with the Breit-Wigner-like strength function $S\left(E_{\gamma}, E_{\mathrm{GDR}}\right)$ defined as

$$
S\left(E_{\gamma}, E_{\mathrm{GDR}}\right)=\frac{1}{\pi} \frac{\gamma_{q}\left(E_{\gamma}\right)}{\left(E_{\gamma}-E_{\mathrm{GDR}}\right)^{2}+\left[\gamma_{q}\left(E_{\gamma}\right)\right]^{2}} .
$$

To compare with the experimental results, the strength function of (22) is employed to calculate the effective GDR strength function in the same way as in Eq. (15), namely

$$
L_{\text {effective }}^{\mathrm{PDM}}\left(E_{\gamma}\right)=\sum_{I, T} P_{p}(I, T) L^{\mathrm{PDM}}\left(E_{\gamma} ; I, T\right) .
$$

Thus, the GDR strength functions were averaged over all possible spins and temperatures with the corresponding values of the GDR strength functions $L^{\mathrm{PDM}}\left(E_{\gamma} ; I, T\right)$ obtained within the PDM for the average masses. The latter were calculated by using the distributions like the ones in Fig. 13. The population matrices obtained from the GEMINI++ presented in Fig. 8, panels (c) and (d), were employed as the weighting factors. The analysis of numerical results carried out within the PDM for ${ }^{88}$ Mo in Refs. [50,51] shows that the GDR width increases with angular momentum at a given value of $T \leqslant 3 \mathrm{MeV}$, whereas it approaches a saturation at $T \simeq 3.5 \mathrm{MeV}$ at $I \geqslant 50 \hbar$. At larger values of $I(I \geqslant 70 \hbar)$ the width saturation takes place at any value of temperature [51]. Pairing is not included in the calculations because its effect is negligible in the range of temperature and angular momentum considered in the present experiment.

\section{RESULTS}

The experimental GDR strength functions were compared to the theoretical predictions of the two previously described models, i.e., the LSD model with thermal shape fluctuations and the PDM, both convoluted with the GEMINI++ population matrices of nuclei participating in the evaporation process; see Fig. 17 for illustration. Moreover, in the thermal shape fluctuation approach the compound nucleus lifetime was also taken into account as it might play an important role for the decaying nuclei at the high-temperature limit.

As it can be seen from the comparisons, in general, the effective GDR strength functions predicted by both the LSD model with TFM and the PDM are found in rather good agreement with the experimental data, especially when the highest energies are concerned. For the lower energy both approaches produce a slightly narrower GDR strength function as compared to the experimental one.

In the case of the thermal shape fluctuation method based on the LSD model, one can observe that the inclusion in the intrinsic width $\Gamma_{0}$ the contribution related to the lifetime of the decaying nucleus, i.e., $\Gamma_{0} \rightarrow \Gamma_{0}(T)=5 \mathrm{MeV}+2 \Gamma_{\mathrm{EV}}$, has almost no effect at the energies corresponding to the lowest considered beam energy of $300 \mathrm{MeV}$. To the contrary, this effect becomes pronounced at the highest bombarding energy of $600 \mathrm{MeV}$, and improves the comparison with the experiment. The rather small effect in the changing of shape of the GDR strength function while including $\Gamma_{\mathrm{EV}}$ is related to the fact that the effective GDR strength function comes from integrating over a wide range of temperature. The possible deviations at the lower bombarding energy might be related to the assumption that the temperature is constant in the shape fluctuation method. Another possible reason could be that the parameters of the LSD model, on which this modeling is based, had no dependence on temperature included.

In the case of the effective GDR strength function obtained within the PDM, one can observe that in general the highenergy tail is well reproduced, both for 300 and $600 \mathrm{MeV}$ data sets. Some deviations observed at low-energy tail of the GDR strength functions could perhaps be explained by the Coriolis splitting, which, even though expected to be low, is not included in the PDM calculations.

The effective GDR widths represented as the FWHM of the experimental GDR strength functions are shown in Fig. 18, with the vertical error bars and horizontal shaded areas indicating the temperature region spanned by the experiment. These experimental values were found within the error bars to be approximately the same, slightly increasing from 9.9(7) MeV for the data corresponding to an average temperature of $2 \mathrm{MeV}$ to $10.3(9) \mathrm{MeV}$ at $3.1 \mathrm{MeV}$.

The rather small dependence of the GDR width on temperature found in this work is in contrast to the much stronger dependence deduced for the ${ }^{132} \mathrm{Ce}$ nucleus [12]. A part of this "saturation" effect can be associated with much higher (almost twice) rotational frequency for the same spin in ${ }^{88} \mathrm{Mo}$ than in ${ }^{132} \mathrm{Ce}$. As a consequence, the GDR width in ${ }^{88} \mathrm{Mo}$ is governed mainly by deformation effects induced by rotation (splitting of the GDR components), which are similar at both considered temperatures, and not so much affected by temperature effects as in the case of ${ }^{132} \mathrm{Ce}$.

In addition, the comparison to the model predictions of the effective GDR widths, based on the LSD model with thermal shape fluctuations (triangles) and based on the PDM (circles) is shown; the lines are plotted to guide the eyes. It should be noted that the increase (if any) of the experimental effective GDR width between $T=2$ and $3.1 \mathrm{MeV}$ has a smaller slope 

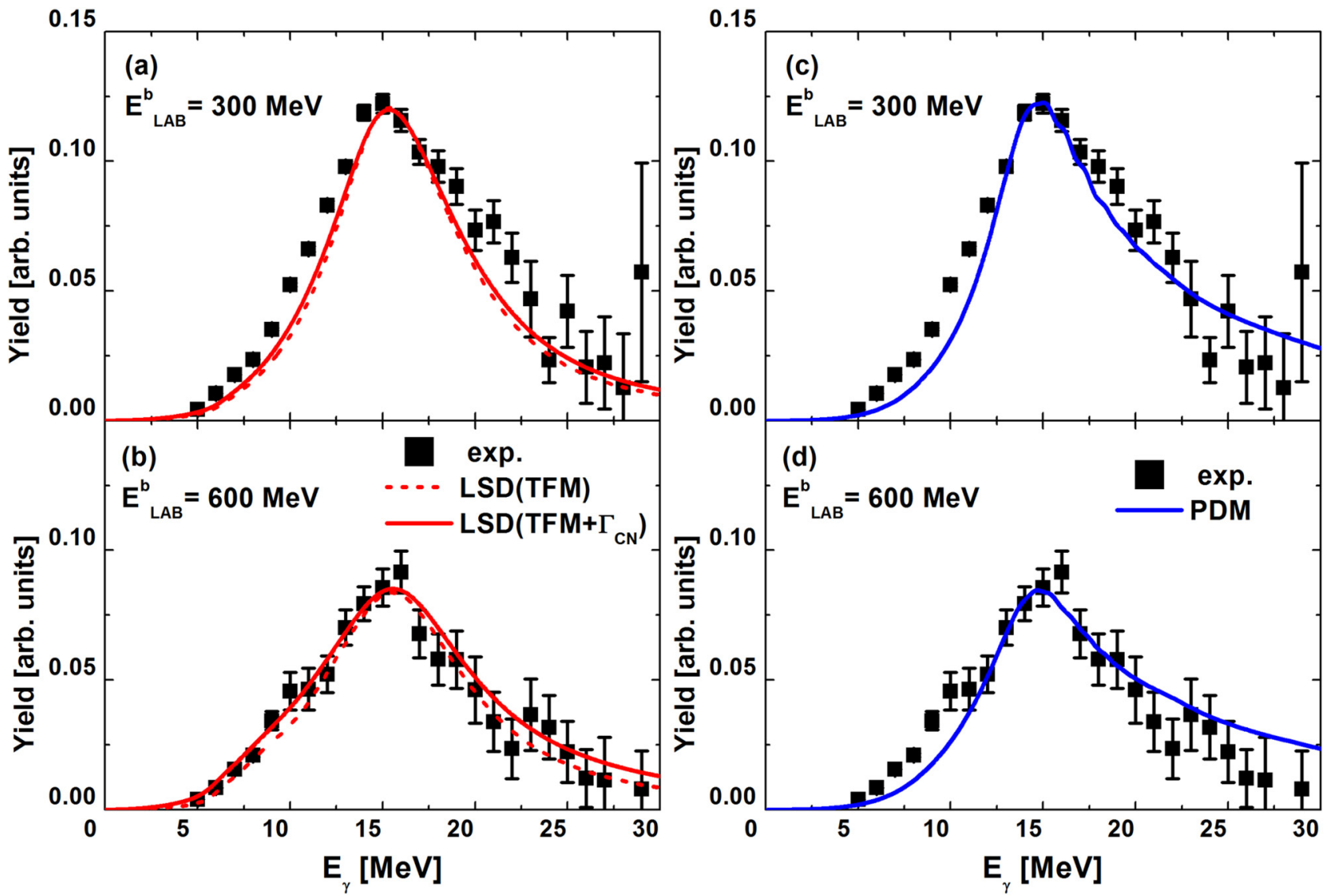

FIG. 17. (Color online) Left: Comparison of the GDR strength functions extracted from the experimental data and the effective model values based on the macroscopic LSD model with TFM [Eq. (15)], for the beam energies of (a) $300 \mathrm{MeV}$ and (b) $600 \mathrm{MeV}$. The theoretical GDR strength functions are estimated with a constant (dashed line) and a temperature-dependent (solid line) intrinsic GDR width $\Gamma_{0}$. Right: Analogous to the left panel comparison of experimentally extracted GDR strength functions to the effective model values based on the PDM [Eq. (24)] for beam energies of (c) $300 \mathrm{MeV}$ and (d) $600 \mathrm{MeV}$.

than the ones predicted by both models. This might indicate the observation of the onset of the GDR width saturation at $T$ around $3 \mathrm{MeV}$. This might be the second reason for observed different behavior of the GDR width than in ${ }^{132} \mathrm{Ce}$. However, because of the wide temperature interval corresponding to each measurement, which is intrinsically related to the evaporation process at high excitation energies, this statement needs further confirmation by additional measurements (for example differential ones; see [52]), where the uncertainty caused by the broad temperature distribution is possibly reduced.

The indication of the onset of the GDR width saturation at high temperatures is further supported by examining the presently measured GDR widths for ${ }^{88} \mathrm{Mo}$ together with the previously measured values for ${ }^{86} \mathrm{Mo}$ [10], ${ }^{92}$ Mo [11], and ${ }^{100}$ Mo [11] at lower temperatures; see Fig. 19. It should be noted that the data available for other Mo isotopes were obtained for different angular momentum distributions, hence with different average angular momentum value and also without the detection of the recoiling nucleus in coincidence. Therefore, to compare these data with the ones of the present experiment, only the data points associated with the mean angular momentum in the interval between $18 \hbar$ and $25 \hbar$ (which is close to the experimental data for ${ }^{88} \mathrm{Mo}$ ) were considered. The temperature for the previous experimental data was obtained similarly as in our case, including GDR emission at different $\mathrm{CN}$ decay steps.

The experimental data are compared with predictions obtained using the phenomenological formula proposed in [53], based on the experimental systematics known at that time. The calculations were performed with the nuclear masses ranging from $80\left({ }^{80} \mathrm{Kr}\right)$ to $100\left({ }^{100} \mathrm{Mo}\right)$. The nucleus ${ }^{80} \mathrm{Kr}$ was chosen because its $A$ and $Z$ correspond to mean $A$ and $Z$ of nuclei emitting GDR $\gamma$ rays in the decay of ${ }^{88} \mathrm{Mo}$, produced at an excitation energy of $261 \mathrm{MeV}$, as calculated by GEMINI++ (see Fig. 13 and Table II ). The angular momentum interval was selected corresponding to the measured data and being close to the value determined for the decay of ${ }^{88} \mathrm{Mo}$. The average values of the angular momentum distributions obtained from the GEMINI++ calculations for the ${ }^{88}$ Mo nuclei decaying by GDR emission were $24 \hbar$ for the $300 \mathrm{MeV}$ and $20 \hbar$ for the $600 \mathrm{MeV}$ case.

Figure 19 shows that the experimental results are quite well described by the calculations performed using the phenomenological function, within about $2 \mathrm{MeV}$ uncertainty. The difference at $3.1 \mathrm{MeV}$ could be due to the fact that parameters used in this formula were deduced using the experimental 


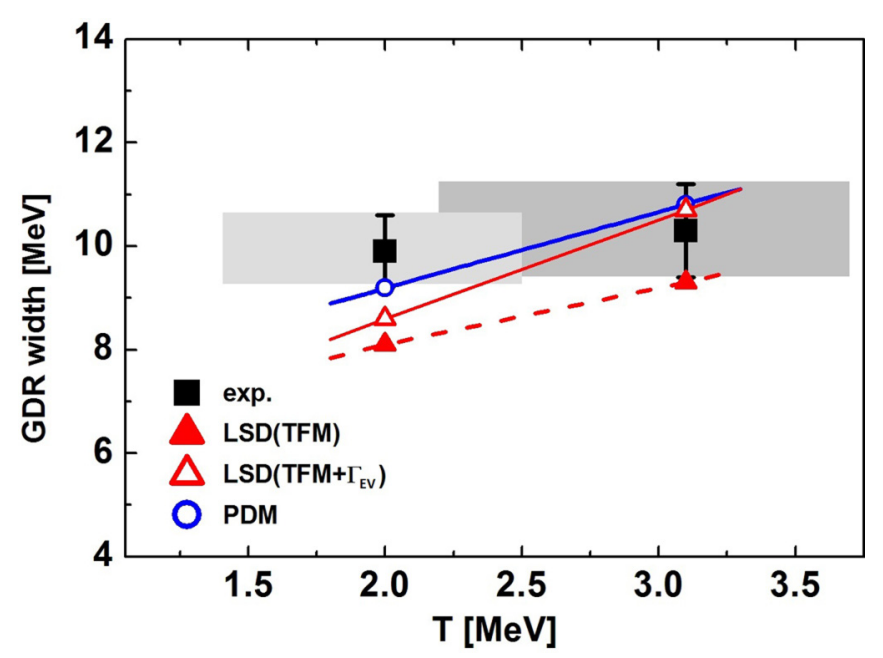

FIG. 18. (Color online) Temperature dependence of the GDR width obtained as the FWHM of the strength functions. The experimental values (full squares) are shown together with the model predictions by the LSD model with TFM (triangles) and the PDM (circles). The results of the calculations based on the LSD model with TFM by using either $\Gamma_{0}=5 \mathrm{MeV}$ (full triangles) or $\Gamma_{0}(T)$ including the lifetimes of the compound nucleus and of nuclei produced in the evaporation process (open triangles) are presented. The experimentally extracted GDR widths are bounded in the shaded areas determined by the width of the experimental temperature distributions and the vertical error bars. The straight lines connecting the theoretical predictions are drawn to guide the eyes.

data available several years ago and not covering the hightemperature region. Specifically, a possible mechanism of the GDR width saturation was not incorporated in the estimates of the parameters of the phenomenological function. Therefore

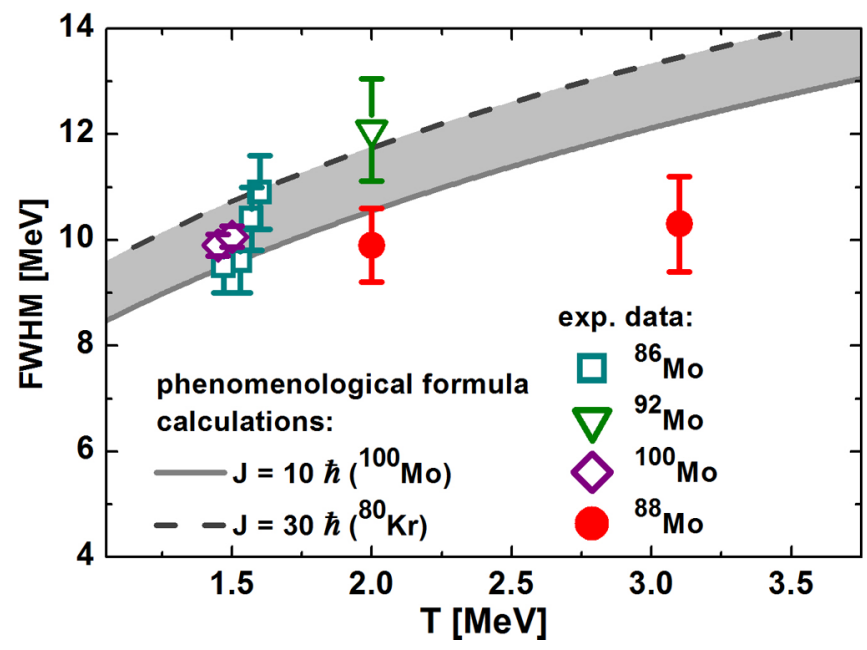

FIG. 19. (Color online) Measured GDR widths as a function of temperature for nuclei in the mass region of ${ }^{88} \mathrm{Mo}$ (full circles) compared with predictions obtained using the expression proposed in [53] based on the experimental systematics (open symbols). The sets of data are selected to correspond to the angular momentum interval $(18-25) \hbar$. The data have the following origins: ${ }^{86} \mathrm{Mo}$ from [10], ${ }^{92}$ Mo from [54], and ${ }^{100}$ Mo from [11]. the indication of the onset of the GDR width saturation in the present article is additionally supported.

\section{SUMMARY AND CONCLUSIONS}

New experimental results for the GDR width of the ${ }^{88} \mathrm{Mo}$ compound nucleus up to the temperature of about $3 \mathrm{MeV}$ were obtained. To extract the GDR width from the experimental data, the statistical-model Monte Carlo GEMINI++ code with the GDR emission incorporated for the first time was employed.

Model calculations were performed using, on the one hand, the thermal shape fluctuation method based on the LSD model and, on the other hand, using the PDM. The resulting GDR strength functions were convoluted, for the first time, with the population matrices of the evaporation process from the GEMINI++ code. Both modeled effective GDR strength functions predict an increase of the GDR width for ${ }^{88} \mathrm{Mo}$ in the investigated temperature region, whereas the experimental data seem to show a weaker increase, although the error bars do not allow for a firm conclusion.

Another element worth emphasizing in relation with the mean-field theory and the description of the collective motion in nuclei has to do with a differentiation between the static, as opposed to the dynamical treatment of the nuclear deformation. More precisely, because of the quantum nature of the nuclear collective motion, the effective deformations are not those corresponding to the static minimum points on the nuclear total potential energy surfaces (like the ones calculated with the help of the LSD model). Indeed, the more profound physical interpretation should be attributed to the most probable deformations and related shapes calculated with the help of the solutions of the collective Schrödinger equation. The first analysis of this type has been performed in Ref. [55], showing systematic differences between the static and the most probable nuclear deformations. An analogous analysis for the nuclei of interest here is in progress and will be published elsewhere.

Moreover, the experimental dependence of the GDR width on the nuclear temperature for the Mo isotopes is, in general, consistent with the systematics expressed by the phenomenological formula. In addition, some indications coming both from the comparison with other experimental data and from the comparison with theoretical predictions, may suggest an onset of saturation of the GDR width at around the temperature of $3 \mathrm{MeV}$, originating both from rotational and temperature effects.

\section{ACKNOWLEDGMENTS}

The authors wish to express their thanks to R. J. Charity and D. Mancusi for valuable discussions related to the GEMINI++ code. This work was supported by the Polish Ministry of Science and Higher Education, Grants No. N N202 486339, No. 2011/03/B/ST2/01894, and No. 2013/08/M/ST2/00591 and by the French-Polish agreements IN2P3-COPIN (Projects No. 06-126, No. 05-119, No. 09-136, and No. 12-145). The numerical calculations within the PDM were carried out using the RIKEN Integrated Cluster of Clusters (RICC) system. 
[1] P. F. Bortignon, A. Bracco, and R. A. Broglia, Giant Resonances: Nuclear Structure at Finite Temperature (Harwood Academic, Amsterdam, 1998).

[2] M. Gallardo et al., Damping of the giant dipole resonance in hot, strongly rotating nuclei, Nucl. Phys. A 443, 415 (1985).

[3] F. Camera et al., Large effects of orientation fluctuations in the angular distribution of the GDR photons, Nucl. Phys. A 572 , 401 (1994).

[4] M. Mattiuzzi et al., Thermal fluctuation and collisional damping effects in the GDR observables in hot rotating ${ }^{176} \mathrm{~W}$ nuclei, Phys. Lett. B 364, 13 (1995).

[5] M. Kmiecik et al., The GDR width in the excited ${ }^{147} \mathrm{Eu}$ compound nucleus at high angular momentum, Nucl. Phys. A 674, 29 (2000).

[6] M. Kmiecik et al., Probing nuclear shapes close to the fission limit with the giant dipole resonance in ${ }^{216} \mathrm{Rn}$, Phys. Rev. C 70, 064317 (2004).

[7] D. Kusnezov and E. Ormand, Giant-dipole resonance and the deformation of hot, rotating nuclei, Phys. Rev. Lett. 90, 042501 (2003).

[8] S. Mukhopadhyay et al., Measurement of giant dipole resonance width at low temperature: A new experimental perspective, Phys. Lett. B 709, 9 (2012).

[9] D. Pandit et al., Critical behavior in the variation of GDR width at low temperature, Phys. Lett. B 713, 434 (2012).

[10] S. K. Rathi et al., Angular momentum gated giant dipole resonance measurements in the reaction ${ }^{28} \mathrm{Si}+{ }^{58} \mathrm{Ni}$ at $E\left({ }^{28} \mathrm{Si}\right)=$ 100 and $125 \mathrm{MeV}$, Phys. Rev. C 67, 024603 (2003).

[11] M. Kicińska-Habior et al., Comparison of giant dipole resonance decay in stiff ${ }^{92}$ Mo and soft ${ }^{100}$ Mo excited nuclei, Phys. Rev. C 45, 569 (1992).

[12] O. Wieland et al., Giant dipole resonance in the hot and thermalized ${ }^{132} \mathrm{Ce}$ nucleus: damping of collective modes at finite temperatures, Phys. Rev. Lett. 97, 012501 (2006).

[13] A. Corsi et al., Excitation of the dynamical dipole in the charge asymmetric reaction ${ }^{16} \mathrm{O}+{ }^{116} \mathrm{Sn}$, Phys. Lett. B 679, 197 (2009).

[14] A. Corsi et al., Measurement of isospin mixing at a finite temperature in ${ }^{80} \mathrm{Zr}$ via giant dipole resonance decay, Phys. Rev. C 84, 041304 (2011).

[15] R. Bass, Fusion of heavy nuclei in a classical model, Nucl. Phys. A 231, 45 (1974).

[16] R. Bass, Nucleus-nucleus potential deduced from experimental fusion cross sections, Phys. Rev. Lett. 39, 265 (1977).

[17] F. Gramegna et al., A telescope with microstrip gas chambers for the detection of charged products in heavy-ion reactions, Nucl. Instrum. Methods A 389, 474 (1997).

[18] M. Bruno et al., GARFIELD + RCo digital upgrade: A modern set-up for mass and charge identification of heavy-ion reaction products, Eur. Phys. J. A 49, 128 (2013).

[19] A. Maj et al., Angular distribution of photons from the decay of the GDR in hot and rotating light $\mathrm{Yb}$ nuclei from exclusive experiments, Nucl. Phys. A 571, 185 (1994).

[20] M. Bini et al., Fiasco: a multidetector optimized for semiperipheral heavy ion collisions at Fermi energies, Nucl. Instrum. Methods A 515, 497 (2003).

[21] R. J. Charity, Systematic description of evaporation spectra for light and heavy compound nuclei, Phys. Rev. C 82, 014610 (2010).

[22] S. Valdré et al. (unpublished).
[23] S. Valdré et al., Measurement of light charged particles in the decay channels of medium-mass excited compound nuclei, EPJ Web Conf. 66, 03090 (2014).

[24] V. L. Kravchuk et al., Light particle emission mechanisms in heavy-ion reactions at 5-20 MeV/u, EPJ Web Conf. 2, 10006 (2010).

[25] M. Ciemała et al., Gamma-decay of the GDR in the GEMINI++ Code, Acta Phys. Pol. B 44, 611 (2013).

[26] W. Hauser and H. Feshbach, The inelastic scattering of neutrons, Phys. Rev. 87, 366 (1952).

[27] P. Möller, J. R. Nix, W. D. Myers and W. J. Swiatecki, Nuclear ground-state masses and deformations, At. Data Nucl. Data Tables 59, 185 (1995).

[28] D. Mancusi (private communication).

[29] M. Kicińska-Habior et al., Search for a phase transition in the nuclear shape at finite temperature and rapid rotation, Phys. Lett. B 308, 225 (1993).

[30] J. J. Gaardhøje et al., Limits of collective motion in hot nuclear matter, Phys. Rev. Lett. 59, 1409 (1987).

[31] J. H. Faou et al., Towards limiting temperatures in nuclei: The behavior of collective motion, Phys. Rev. Lett. 72, 3321 (1994).

[32] D. Santonocito et al., Onset of quenching of the giant dipole resonance at high excitation energies, Phys. Rev. C 90, 054603 (2014).

[33] K. Pomorski, J. Dudek, Nuclear liquid-drop model and surfacecurvature effects, Phys. Rev. C 67, 044316 (2003).

[34] J. Dudek, K. Pomorski, N. Schunck and N. Dubray, Hyperdeformed and megadeformed nuclei: Lessons from the slow progress and emerging new strategies, Eur. Phys. J. A 20, 15 (2004).

[35] N. Dubray, J. Dudek, and A. Maj, Statistical description of the thermal shape fluctuations using realistic microscopic and macroscopic models, Acta Phys. Pol. B 36, 1161 (2005).

[36] N. Dinh Dang and A. Arima, Quantal and thermal dampings of giant dipole resonances in ${ }^{90} \mathrm{Zr}$, ${ }^{120} \mathrm{Sn}$ and ${ }^{208} \mathrm{~Pb}$, Phys. Rev. Lett. 80, 4145 (1998); Temperature dependence of quantal and thermal dampings of the hot giant dipole resonance, Nucl. Phys. A 636, 427 (1998).

[37] W. E. Ormand, P. F. Bortignon, and R. A. Broglia, Effects of angular momentum projection on the nuclear partition function and the observation of the giant-dipole resonance in hot nuclei, Nucl. Phys. A 618, 20 (1997).

[38] Y. Alhassid and N. Whelan, The Jacobi transition and the giantdipole resonance in rapidly rotating hot nuclei, Nucl. Phys. A 565, 427 (1993).

[39] M. Kmiecik et al., GDR feeding of the highly-deformed band in ${ }^{42} \mathrm{Ca}$, Acta Phys. Pol. B 36, 1169 (2005).

[40] K. Mazurek, N. Dubray, J. Dudek and N. Schunck, Exotic deformations in the actinide region, Int. J. Mod. Phys. E 15, 542 (2006).

[41] K. Mazurek, M. Matejska, M. Kmiecik, A. Maj and J. Dudek, Influence of the level density parametrization on the effective GDR width at high spins, Int. J. Mod. Phys. E 17, 132 (2008).

[42] M. Brekiesz et al., Study of deformation effects in the charged particle emission from ${ }^{46} \mathrm{Ti}$, Nucl. Phys. A 788, 224c (2007).

[43] M. Kmiecik et al., Strong deformation effects in hot rotating ${ }^{46}$ Ti, Acta Phys. Pol. B 38, 1437 (2007).

[44] J. Dudek, Z. Szymański, and T. R. Werner, Woods-Saxon potential parameters optimized to the high spin spectra in the lead region, Phys. Rev. C 23, 920 (1981); see also J. Dudek et al., 
Description of the high spin states in ${ }^{146} \mathrm{Gd}$ using the optimized Woods-Saxon potential, ibid. 26, 1712 (1982); S. Kahane, S. Raman, and J. Dudek, Testing the parameters of the "Universal" Woods-Saxon potential with $B(E 2 ; 0+1 \rightarrow 2+1)$ values and nucleon separation energies, ibid. 40, 2282 (1989).

[45] K. Nergaard, The isovector dipole mode in heated rotating nuclei, Phys. Lett. B 110, 7 (1982).

[46] J. J. Gaardhøje et al., Nuclear structure at high excitation energy studied with giant resonances, Annu. Rev. Nucl. Part. Sci. 42, 483 (1992).

[47] J. J. Gaardhøje et al., Atomic nuclei at high excitation energy studied with giant resonances, Acta Phys. Pol. B 24, 139 (1993).

[48] P. Chomaz, Influence of the compound-nucleus lifetime on the observed width of hot giant dipole resonances, Phys. Lett. B 347, 1 (1995).

[49] N. Dinh Dang and A. Arima, Pairing effect on the giant dipole resonance width at low temperature, Phys. Rev. C 68, 044303 (2003); N. Dinh Dang and N. Quang Hung, Giant dipole resonance in ${ }^{201} \mathrm{Tl}$ at low temperature, ibid. 86, 044333 (2012); B. Dey et al., Probing the critical behavior in the evolution of
GDR width at very low temperatures in $A \sim 100$ mass region, Phys. Lett. B 731, 92 (2014).

[50] N. Dinh Dang, Damping of giant dipole resonances in hot rotating nuclei, Phys. Rev. C 85, 064323 (2012).

[51] N. Dinh Dang, M. Ciemala, M. Kmiecik and A. Maj, Giant dipole resonance in ${ }^{88} \mathrm{Mo}$ from phonon damping model strength functions averaged over temperature and angular momentum distributions, Phys. Rev. C 87, 054313 (2013).

[52] A. Maj et al., Phase-space selection of the GDR $\gamma$ ray emission from hot ${ }^{162}$ Yb Nuclei, Phys. Lett. B 291, 385 (1992).

[53] D. Kusnezov, Y. Alhassid, and K. A. Snover, Scaling properties of the giant dipole resonance width in hot rotating nuclei, Phys. Rev. Lett. 81, 542 (1998).

[54] J. H. Gundlach et al., Oblate deformed shapes of hot rotating nuclei deduced from giant-dipole-resonance decay studies, Phys Rev. Lett. 65, 2523 (1990).

[55] K. Mazurek, J. Dudek, A. Maj, and D. Rouvel, Nuclear Jacobi and Poincaré transitions at high spins and temperatures: Account of dynamic effects and large-amplitude motion, Phys. Rev. C 91, 034301 (2015). 